\title{
HOLOMORPHIC EXTENSION OF CR FUNCTIONS FROM QUADRATIC CONES
}

\author{
DEBRAJ CHAKRABARTI AND RASUL SHAFIKOV
}

\begin{abstract}
It is proved that CR functions on a quadratic cone $M$ in $\mathbb{C}^{n}, n>1$, admit one-sided holomorphic extension whenever $M$ does not have two-sided support, a geometric condition on $M$ which generalizes minimality in the sense of Tumanov. A biholomorphic classification of quadratic cones in $\mathbb{C}^{2}$ is also given.
\end{abstract}

\section{INTRODUCTION.}

One of the central results in the theory of CR functions is Trépreau's theorem 21 on local holomorphic extension of CR functions defined on a smooth real hypersurface $M$ in $\mathbb{C}^{n}$ to one side of $M$. Its generalization [22, 2] to the case when $M$ is of higher codimension is known as wedge-extendability. In both cases simultaneous extension of all CR functions to an open set in $\mathbb{C}^{n}$ adjacent to $M$ is equivalent to minimality of $M$. In the hypersurface case this simply means that $M$ does not contain any germ of complex analytic hypersurface. For more details on this subject see the recent extensive survey [17] and the references there.

CR functions can be defined on a wider class of objects than smooth CR submanifolds, for example on locally Lipschitz graphs (see e.g., [11). It is therefore natural to study the properties of CR functions defined on non-smooth objects, in particular, a natural question to ask is whether a similar one-sided holomorphic extension of CR functions holds when the hypersurface $M$ is no longer smooth. More generally, one can ask whether all functions holomorphic on one side of $M$ extend holomorphically to the other side near non-smooth points of $M$. The answer is affirmative if $M$ is the graph of a continuous function, as proved in [10. While the problem could be very difficult in general, it seems that the situation when $M$ is a real analytic variety is of particular interest because of the connection with questions related to boundary regularity and analytic continuation of holomorphic mappings between domains with real analytic boundary.

In this paper we consider the family of real quadratic cones in $\mathbb{C}^{n}, n>1$, and prove a Trépreau-type extension result for $\mathrm{CR}$ functions. By a (real) quadratic cone we mean an irreducible real algebraic set $M$ in $\mathbb{C}^{n}$ of pure dimension $2 n-1$ defined by

$$
M=\left\{z \in \mathbb{C}^{n}: \rho(z)=0\right\},
$$

where $\rho(z)$ is a real-valued homogeneous polynomial of degree two in $x_{j}$ and $y_{j}$, where $z_{j}=x_{j}+i y_{j}, 1 \leq j \leq n$, are the coordinates on $\mathbb{C}^{n}$. These cones are perhaps the simplest examples of real analytic varieties with singularities, and they form a natural class of models to study. Let $M^{\text {reg }}$ be the smooth part of $M$. We call $f \in L_{\text {loc }}^{1}(M)$ a CR function, if $\int_{M^{\text {reg }}} f \bar{\partial} \varphi=0$ for every smooth $(n, n-2)-$ form $\varphi$ with compact support in $\mathbb{C}^{n}$. In particular, every continuous function on $M$, which 
is $\mathrm{CR}$ on $M^{\mathrm{reg}}$, is $\mathrm{CR}$ on $M$ (see Lemma 3.1 below.) We refer to Sections 2 and 3 for further discussion concerning quadratic cones and CR functions. It turns out that minimality is no longer a sufficient condition for one-sided extension. Indeed, consider

$$
M=\left\{z=\left(z_{1}, z_{2}\right) \in \mathbb{C}^{2}: \rho(z)=\operatorname{Re}\left(\frac{1}{2} z_{1}^{2}+\frac{1}{3} z_{2}^{2}\right)+\left|z_{1}\right|^{2}-\left|z_{2}\right|^{2}=0\right\} .
$$

Then $M$ is a quadratic cone of dimension three, in fact, $M$ is a smooth minimal manifold away from the origin, and $\mathbb{C}^{2} \backslash M$ consists of two connected components $\Omega^{ \pm}=\left\{z \in \mathbb{C}^{2}: \pm \rho(z)>0\right\}$. Consider the function

$$
f(z)=\frac{z_{2}^{2}}{z_{1}}-\frac{z_{1}^{2}}{z_{2}}
$$

By letting $f(0)=0$, it is easy to see that $\left.f\right|_{M}$, the restriction of $f$ to $M$, extends continuously to the origin, and therefore $\left.f\right|_{M}$ is a continuous CR function. Like CR functions on smooth hypersurfaces, $f$ admits the jump representation $f=F^{+}-F^{-}$, where $F^{+}=\frac{z_{2}^{2}}{z_{1}} \in \mathcal{O}\left(\Omega^{+}\right)$and $F^{-}=\frac{z_{1}^{2}}{z_{2}} \in \mathcal{O}\left(\Omega^{-}\right)$. However, since $\left\{z \in \mathbb{C}^{2}: z_{2}=0\right\}$ is contained in $\overline{\Omega^{+}}$, and $\left\{z_{1}=0\right\}$ is contained in $\overline{\Omega^{-}}$, it follows that $\left.f\right|_{M}$ does not extend holomorphically to either side of $M$.

In the above example the obstruction to holomorphic extension is the presence of two complex hypersurfaces $A^{+}=\left\{z_{2}=0\right\}$ and $A^{-}=\left\{z_{1}=0\right\}$ residing on different sides of $M$. We prove that for quadratic cones this is the only obstruction. To formulate this condition we make the following definition. Given a real analytic hypersurface $M$, i.e., a real analytic set of pure dimension $2 n-1$ in $\mathbb{C}^{n}$, we call $\rho(z)$ a defining function of $M$ near point $p \in M$ if $\rho$ is real analytic in a neighbourhood $\Omega$ of $p, M \cap \Omega=\{z \in \Omega: \rho(z)=0\}$, and there exists a point $z \in M \cap \Omega$ such that $d \rho(z) \neq 0$. Clearly, any real analytic hypersurface $M$ admits a defining function at each of its points.

Let $\Omega^{ \pm}=\{z \in \Omega: \pm \rho(z)>0\}$. Then $\Omega^{ \pm}$are non-empty open sets which up to a sign are independent of the choice of the defining function of $M$, as proved in Lemma 2.1 below. We note that in general $\Omega^{ \pm}$may consist of several connected components.

Definition 1.1. Let $M$ be an irreducible real analytic hypersurface in $\mathbb{C}^{n}$. Let $\rho(z)$ be a defining function of $M$ in some neighbourhood $\Omega$ of a point $p \in M$. We say that $M$ admits two-sided support by complex hypersurfaces at $p \in M$ if there exist germs at $p$ of complex analytic hypersurfaces $A^{+}$and $A^{-}$such that $A^{ \pm} \subset \overline{\Omega^{ \pm}}$.

In short we say that $M$ as above has two-sided support at $p$. As in the smooth case, we say that a real analytic hypersurface $M$ is minimal at $p \in M$, if there is no germ of complex analytic hypersurface at $p$ contained in $M$. Note, in particular, that any hypersurface $M$, which is non-minimal at $p \in M$, has two-sided support at $p$.

It is well-known (see 15]) that a smooth pseudoconvex hypersurface need not have a supporting complex hypersurface on the pseudoconcave side, and therefore in general the existence of such support is irrelevant to holomorphic non-extendability of domains in $\mathbb{C}^{n}$. However, in the question of simultaneous analytic continuation of all holomorphic functions from one side of $M$ to the other side, the existence of supporting complex hypersurfaces becomes important. Our first result concerns the extension of holomorphic functions defined on one side of a cone. 
Theorem 1.1. Let $M=\{\rho=0\}$ be a quadratic cone in $\mathbb{C}^{n}, n>1$, where $\rho$ is the defining function of $M$. Given a neighbourhood $\Omega$ of 0 in $\mathbb{C}^{n}$, let $\Omega^{+}$and $\Omega^{-}$be the open sets in $\mathbb{C}^{n}$ defined by $\Omega^{ \pm}=\{z \in \Omega: \pm \rho(z)>0\}$. Then the following are equivalent.

(a) $M$ does not have two-sided support at the origin.

(b) There exists a neighbourhood $U$ of the origin such that either all functions in $\mathcal{O}\left(\Omega^{+}\right)$or all functions in $\mathcal{O}\left(\Omega^{-}\right)$extend holomorphically to $U$.

We note that in the above theorem the singular part of the cone $M$ can have arbitrary dimension. Quadratic cones provide some understanding of singularities of arbitrary analytic sets. We believe that Definition 1.1 is also relevant to extension results for arbitrary real analytic sets. This is the subject of our further investigation.

Combining Theorem 1.1 with the jump formula for CR functions, we obtain the following result.

Corollary 1.1. If $M$ does not have two-sided support at the origin, then there exists a neighbourhood $U$ of the origin such that every $C R$ function on $\Omega \cap M$ has a holomorphic extension either to $U \cap \Omega^{+}$or $U \cap \Omega^{-}$. In particular, if $f$ is a continuous function on $M \cap \Omega$ and $C R$ on $M^{\mathrm{reg}}$, then there is a function $F$, holomorphic in $U \cap \Omega^{+}$or $U \cap \Omega^{-}$and continuous on $M^{\mathrm{reg}} \cap U$, with $\left.F\right|_{M^{\mathrm{reg}} \cap U}=f$.

It remains an open question whether for continuous $f$ the equality $F=f$ holds on the singular part of $M \cap U$. An answer will depend upon a better understanding of boundary behavior of the jump formula, for example as in [18].

We also note here that in the non-smooth case the well-known approximation theorem due to Baouendi and Trèves [3], fails in general. The conditions under which a CR function on a non-smooth hypersurface can be approximated by holomorphic polynomials are not known. In fact, for $M$ given by (2) in the example above, not all CR functions on $M$ can be approximated by polynomials. Indeed, if this were true, then by the maximum principle, approximation by polynomials would also hold on the union of all holomorphic discs attached to $M$ near the origin. Fix some small $\epsilon>0$ and let $D_{\epsilon}=A^{+} \cap\left\{\left|z_{1}\right| \leq \epsilon\right\}$. Then $\partial D_{\epsilon}$ is compactly contained in $\Omega^{+}$. If $\tilde{D}_{\epsilon}$ is a small perturbation of $D_{\epsilon}$, the set $\tilde{D}_{\epsilon} \cap{\overline{\Omega^{-}}}^{-}$is a disc attached to $M$ whenever its interior is nonempty. We can therefore produce a family of holomorphic discs attached to $M$ that sweep a small one-sided neighbourhood of the origin. But this would imply that any CR function can be extended holomorphically to one side of $M$. However, the function in (3) does not admit such extension.

Trépreau's original proof 21] relies on the existence of Bishop disks attached to a smooth hypersurface. Alternatively one can use the technique of propagation of holomorphic extendability along CR orbits (see [17), or Shcherbina's [19] characterization of polynomial hulls of continuous 2 -spheres in $\mathbb{C}^{2}$ (as in [10]). The last technique yields the analog of Trépreau's theorem even for graphs of continuous functions. However, these methods require the hypersurface either to be smooth or locally represented as a graph, which is impossible for quadratic cones near the singular points.

We therefore follow a different route to prove Theorem 1.1. For $n=2$, when $M$ does not have two-sided support, we explicitly construct a family of analytic discs touching $M$ at the origin and then apply the Kontinuitätssatz. A similar technique 
was also used in [4. The case $n>2$ is then reduced to $n=2$ by using biholomorphic invariants of cones, which will be obtained in the proof of Theorem 1.2 below, and a slicing argument.

In order to construct the discs we obtain a complete classification of quadratic cones in $\mathbb{C}^{2}$ under biholomorphic equivalence. Such biholomorphic classification of non-smooth hypersurfaces is of independent interest (see e.g., [5] for classification of non-smooth Levi-flats.)

Theorem 1.2. Let $M$ be a quadratic cone. Then there is a unique normal form $\rho$ in the last column of the table below, such that $M$ is biholomorphic to $\{\rho=0\}$. More precisely, there is a unique type in column 2 of the table determined by the signature of the hermitian part of $\rho$ given in column 1, and a unique choice of parameters in the range given in column 3 so that the germ of $M$ at the origin is biholomorphic to the corresponding germ of $\{\rho=0\}$, where $\rho$ is the defining function in the normal form in the last column of this row with these parameters.

\begin{tabular}{|l|l|c|l|}
\hline$(\pi, \nu)$ & Type & Parameters & Defining Function \\
\hline \multirow{2}{*}{$(2,0)$} & $\mathcal{M}_{(2,0)}$ & $\begin{array}{c}0 \leq B \leq A, \\
A>1\end{array}$ & $\operatorname{Re}\left(A z_{1}^{2}+B z_{2}^{2}\right)+\left|z_{1}\right|^{2}+\left|z_{2}\right|^{2}$ \\
\hline \multirow{5}{*}{$(1,1)$} & $\mathcal{M}_{(1,1)}^{1}$ & $0 \leq B \leq A$ & $\operatorname{Re}\left(A z_{1}^{2}+B z_{2}^{2}\right)+\left|z_{1}\right|^{2}-\left|z_{2}\right|^{2}$ \\
\cline { 2 - 4 } & $\mathcal{M}_{(1,1)}^{2}$ & $\begin{array}{c}\operatorname{Re} A>0, \\
\operatorname{Im} A \geq 0\end{array}$ & $\operatorname{Re}\left(A z_{1}^{2}+\bar{A} z_{2}^{2}\right)+\operatorname{Im}\left(z_{1} \bar{z}_{2}\right)$ \\
\cline { 2 - 4 } & $\mathcal{M}_{(1,1)}^{3}$ & & $\operatorname{Re}\left(z_{1}^{2}\right)+\operatorname{Im}\left(z_{1} \bar{z}_{2}\right)$ \\
\cline { 2 - 4 } & $\mathcal{M}_{(1,1)}^{4}$ & $A>0$ & $\operatorname{Re}\left(z_{1}^{2}+i A z_{1} z_{2}\right)+\operatorname{Im}\left(z_{1} \bar{z}_{2}\right)$ \\
\hline \multirow{3}{*}{$(1,0)$} & $\mathcal{M}_{(1,0)}^{1}$ & $A \geq 0$ & $\operatorname{Re}\left(A z_{1}^{2}+z_{2}^{2}\right)+\left|z_{1}\right|^{2}$ \\
\cline { 2 - 4 } & $\mathcal{M}_{(1,0)}^{2}$ & & $\operatorname{Re}\left(z_{1} z_{2}\right)+\left|z_{1}\right|^{2}$ \\
\hline$(0,0)$ & $\mathcal{M}_{(0,0)}^{1}$ & & $\operatorname{Re}\left(z_{1}^{2}+z_{2}^{2}\right)$ \\
\hline
\end{tabular}

Note: After submitting this article for publication, we came to know of the work of Ermolaev 13, in which real quadratic forms in $\mathbb{C}^{n}$ are classified by an algebraic method very different from ours. However, our method yields directly the invariants required for the proof of Theorem 1.1 for $n \geq 3$.

Two-sided support by complex hypersurfaces is a natural generalization of the notion of non-minimality. The following result is probably well-known, but for the sake of completeness we will give the proof.

Proposition 1.1. Let $M \subset \mathbb{C}^{n}$ be a $\mathcal{C}^{1}$ hypersurface, or more generally, suppose that $M$ can be represented locally as a Lipschitz graph. Let $p \in M$, and let $M$ divide a neighbourhood $\Omega$ of $p$ in $\mathbb{C}^{n}$ into two components $\Omega^{ \pm}$. Suppose that $M$ has two-sided support at $p$, so that there are germs at $p$ of complex hypersurfaces $A^{ \pm} \subset \overline{\Omega^{ \pm}}$. Then $A^{+}=A^{-} \subset M$.

The paper is organized as follows: in Section 2 we discuss properties of quadratic cones, in Section 3 we review the material on CR functions and prove Corollary 1.1. Section 4 contains some preliminary results in Linear Algebra. Theorem 1.2 is proved in Section 5 and Theorem 1.1 in Section 6. As an application of the proof of Theorem 1.1 we classify in Section 7 all quadratic cones that have two-sided support. Finally, Proposition 1.1 is proved in Section 8.

Note on this version: An earlier version of this article was published as [6], and subsequently an erratum 7 correcting some mistakes was published. This version 
incorporates the corrections from [7], as well as corrections of a few minor typos, and is posted in ArXiv.

Acknowledgment. The authors would like to thank A. Boivin, X. Gong, J.-P. Rosay and A. Sukhov for helpful discussions.

\section{Quadratic Cones.}

2.1. Real analytic hypersurfaces have two sides. We say that $M$ is a real analytic subset of a domain $U \subset \mathbb{R}^{N}$ if near each point $p \in U$ it is defined as the zero set of a finite collection of real analytic functions near $p$. $M$ is called irreducible if it cannot be represented as a union of two non-empty real analytic sets. A point $p \in M$ is called regular or smooth of dimension $d$, if near $p, M$ is a $d$-dimensional real analytic manifold. Dimension of $M$ is then defined as the maximum dimension at regular points. We say that $M$ has pure dimension $d$ if all regular points of $M$ have dimension $d$. We will say that $M$ is a real analytic hypersurface in $\mathbb{R}^{N}$ if it is of pure dimension $N-1$. Denote by $M^{\text {reg }}$ the set of regular points of $M$. Then $M^{\text {sng }}=M \backslash M^{\text {reg }}$ is the set of singular points.

Lemma 2.1. Let $M \subset \mathbb{R}^{N}$ be an irreducible real analytic hypersurface which is given in some open set $\Omega$ by the defining function $\rho$ (recall from Section 1 that this means that there is a point $x \in M \cap \Omega$ such that $d \rho(x) \neq 0)$. Let $\Omega^{ \pm}=\{ \pm \rho>0\}$. Then up to a sign, the sets $\Omega^{ \pm}$are independent of the choice of the defining function of $M$.

Proof. Let $S=\{x \in \Omega: d \rho(x)=0\} \cap M$. Then $S$ is a real analytic set of dimension at most $N-2$, in particular, $S$ is nowhere dense in $M$, and $M^{\text {sng }} \subset S$. Suppose $\tilde{\rho}(x)$ is another defining function of $M$, real analytic in $\Omega$, and let $\tilde{S}$ be defined similarly. Consider

$$
V=\{x \in \Omega: \rho(x)>0, \tilde{\rho}(x)<0\} \cup\{x \in \Omega: \rho(x)<0, \tilde{\rho}(x)>0\} .
$$

It is enough to prove that either $\bar{V}=\Omega$ or $V=\varnothing$. Suppose that $V \neq \varnothing$. If $\bar{V} \neq \Omega$, then there exists $q \in(\partial V \cap \Omega) \backslash(S \cup \tilde{S})$. Since both $\rho$ and $\tilde{\rho}$ change the sign near $q$, we conclude that $q$ is an interior point of $\bar{V}$. This shows that $\bar{V}=\Omega$.

2.2. Cones: real and hermitian signatures. Let $M$ be a quadratic cone in $\mathbb{R}^{N}$, i.e., $M=\{\rho=0\}$, where $\rho: \mathbb{R}^{N} \rightarrow \mathbb{R}$ is a homogeneous quadratic polynomial, and $M$ is irreducible of dimension $N-1$. Since the Weierstrass pseudo-polynomial that represents $M$ has degree two, the projection from $M$ to any $N-1$ dimensional linear subspace is always a two-sheeted covering on some open set near the origin. It follows that a quadratic cone can never be represented as the graph of a continuous function.

After a linear change of coordinates on $\mathbb{R}^{N}$, and changing $\rho$ to $-\rho$ if required, we can assume that

$$
\rho(x)=\sum_{0<i \leq p} x_{i}^{2}-\sum_{p<i \leq p+q} x_{i}^{2},
$$

where $(p, q)$ will be called the real signature of $M$ with $p \geq q \geq 1$. ( Note that if $q=0, M$ reduces to a point, and if $p=q=1, M$ is reducible.) The real geometry of cones with $q=1$ and $q>1$ show certain differences: if $q>1$, the set $\mathbb{R}^{N} \backslash M$ has two components, and $M^{\text {reg }}$ is connected. If $q=1$, then $\mathbb{R}^{N} \backslash M$ has three components. If $\rho$ is given by (5), and $\Omega^{ \pm}$are as in Lemma 2.1, the set $\Omega^{-}$consists 
of two components whereas the set $\Omega^{+}$is connected. Also, $M^{\text {reg }}$ is disconnected and has two components.

We now consider real quadratic cones in complex space. Let $\rho(z)$ be a real-valued homogeneous polynomial of degree two in $\mathbb{C}^{n}$, and let $M$ be a quadratic cone given by (1). We associate with $M$ a pair of non-negative integers $(\pi, \nu)$, where $\pi$ (resp. $\nu)$ is the number of positive (resp. negative) eigenvalues of the hermitian form

$$
h_{\rho}(z, w)=\sum_{i, j=1}^{n} \frac{\partial^{2} \rho}{\partial \overline{z_{i}} \partial z_{j}} \overline{z_{i}} w_{j}=\bar{z}^{t} H w,
$$

where $A^{t}$ denotes the transpose of a matrix $A$. We call $(\pi, \nu)$ the hermitian signature of $M$. Since $\rho=0$ and $-\rho=0$ define the same cone $M$, we can always assume that $\pi \geq \nu$.

Let $t_{\rho}(z)=\rho(z)-h_{\rho}(z, z)$. Then for each $i, j$,

$$
\frac{\partial^{2} t_{\rho}}{\partial \overline{z_{i}} \partial z_{j}} \equiv 0
$$

i.e., $t_{\rho}$ is a real pluriharmonic homogeneous polynomial of degree two in $\mathbb{C}^{n}$. There exists a holomorphic homogeneous polynomial $s_{\rho}$ of degree two in $\mathbb{C}^{n}$, such that $t_{\rho}(z)=\operatorname{Re}\left(s_{\rho}\right)$, and therefore,

$$
\rho(z)=\operatorname{Re}\left(z^{t} S z\right)+\bar{z}^{t} H z
$$

where $S \in \operatorname{Sym}(n, \mathbb{C})$, the space of symmetric complex matrices, and $H$ is an $n \times n$ hermitian matrix. We refer to $\operatorname{Re}\left(z^{t} S z\right)$ and $\bar{z}^{t} H z$ as the harmonic and the hermitian parts of the form $\rho$ respectively.

2.3. Tangent cones. For a set $E \subset \mathbb{R}^{n}$, with $0 \in E$, the tangent cone $T_{0} E$ to $E$ at the origin is defined as the set of all limit vectors $v \in \mathbb{R}^{n}$ for all sequences of vectors of the form $t_{j} a_{j}$, where $a_{j} \in E, \lim _{j \rightarrow \infty} a_{j}=0$, and $t_{j}>0$. Clearly, if $E$ is a smooth manifold near the origin, then $T_{0} E$ coincides with the tangent plane to $E$ at the origin (hence the notation). In general, $T_{0} E$ is a real cone in the sense that $t v \in T_{0} E$ for $v \in T_{0} E$, and $t>0$. We note that if $M$ is a real quadratic cone in $\mathbb{R}^{n}$ (or $\mathbb{C}^{n}$ ), then $T_{0} M=M$. Indeed, if $a \in M$, then ta $\in M$, and therefore, $t a \in T_{0} M$ for all $t \in \mathbb{R}$. In particular, $a \in T_{0} M$. On the other hand, if $a \in T_{0} M$, then there exists a sequence of point $a_{j} \in M, a_{j} \rightarrow 0$, and $t_{j}>0$ such that $a=\lim _{j \rightarrow \infty} t_{j} a_{j}=a$. Since $t_{j} a_{j} \in M$, and $M$ is closed, we conclude that $a \in M$.

Lemma 2.2. Let $M, M^{\prime} \subset \mathbb{C}^{n}$ be two germs of quadratic cones at 0 which are biholomorphic. Then we can take the biholomorphism to be a complex linear map.

Proof. Let $F$ be a biholomorphic map from $M$ to $M^{\prime}$. Then by [9, Prop.1, Sec. 8.2], $d F_{0}\left(T_{0} M\right)=T_{0} M^{\prime}$, where $d F_{0}$ is the differential of $F$ at 0 . Since $M=T_{0} M$ and $M^{\prime}=T_{0} M^{\prime}$, we see that $M, M^{\prime}$ are in fact linearly biholomorphic.

It follows that the real and hermitian signatures are not only linear but in fact biholomorphic invariants of germs of quadratic cones at the origin. 


\section{CR MANIFOLDS AND FUNCTIONS.}

3.1. Definitions and examples. Recall that an (embedded) CR manifold is a $\left(\mathcal{C}^{1}-\right)$ smooth manifold in $\mathbb{C}^{n}$ such that the dimension of $H_{p} M=T_{p} M \cap i T_{p} M$ is independent of $p \in M$. The (complex) dimension of $H_{p} M$ is then called the $\mathrm{CR}$ dimension of $M$. In particular, any smooth real hypersurface in $\mathbb{C}^{n}$ is a $\mathrm{CR}$ manifold of CR dimension $n-1$.

Let $M$ be a smooth real hypersurface in $\mathbb{C}^{n}$. Recall that a distribution $f$ on $M$ is said to be $C R$ if it satisfies the tangential Cauchy-Riemann equations. If further $f \in L_{\text {loc }}^{1}(M)$, and $M$ is orientable, this means that for any $C^{\infty}$-smooth $(n, n-2)$-form $\varphi$ with compact support on $M$ one has

$$
\int_{M} f \bar{\partial} \varphi=0
$$

Suppose now that $M$ is an irreducible real analytic hypersurface in some domain $\Omega$ in $\mathbb{C}^{n}$. Near every $p \in M^{\text {reg }}, M$ is a $\mathrm{CR}$ manifold of $\mathrm{CR}$ dimension $n-1$. Furthermore (see e.g [14]), $M$ defines a degree one current on $\Omega$, denoted by $[M]$, which acts on a compactly supported test form $\varphi$ of degree $2 n-1$ on $\Omega$ by

$$
[M](\varphi)=\int_{M^{\mathrm{reg}}} \varphi .
$$

We write $[M]=[M]^{0,1}+[M]^{1,0}$ for the natural splitting of $[M]$ into a sum of currents of bidegree $(0,1)$ and $(1,0)$. Let $d \lambda_{2 n-1}$ denote the $(2 n-1)$-dimensional Lebesgue measure on $M^{\mathrm{reg}}$. We say that $f \in L_{\mathrm{loc}}^{1}(M)$, if for any compact set $K \subset \Omega$, we have $\int_{M^{\mathrm{reg}} \cap K}|f| d \lambda_{2 n-1}<\infty$. Then $f[M], f[M]^{0,1}$ and $f[M]^{1,0}$ are well-defined currents. If $M^{\text {reg }}=M$, then (7) simply means that $f$ is CR whenever the current $f[M]^{0,1}$ is $\bar{\partial}$-closed in $\Omega$. This leads to the following

Definition 3.1. Let $M$ be a real analytic hypersurface (possibly with singularities) in a domain $\Omega \subset \mathbb{C}^{n}$, and $f \in L_{\mathrm{loc}}^{1}(M)$. Then $f$ is called $C R$ if for any $C^{\infty}$-smooth $(n, n-2)$-form $\varphi$ with compact support on $\Omega$,

$$
\int_{M^{\mathrm{reg}}} f \bar{\partial} \varphi=0
$$

or, equivalently, if $\bar{\partial}\left(f[M]^{0,1}\right)=0$.

It is important to note that according to this definition, a function $f$ in $L_{\text {loc }}^{1}(M)$, which is $\mathrm{CR}$ on $M^{\mathrm{reg}}$, is not necessarily $\mathrm{CR}$ on $M$, even when $M^{\text {sng }}$ is a single point, see e.g., [16, Example 2.2]. In other words, the singularity of $M$ is not in general CR-removable for CR functions on $M^{\text {reg }}$. However, if we assume further conditions regarding the growth of $f$ near $M^{\text {sng }}$, we may conclude that $f$ is $\mathrm{CR}$ on $M$. The following result is also proved in [16]. Let $M=\{\rho(z)=0\}$ be a real analytic hypersurface, and suppose that $\sigma=M \cap\{d \rho=0\}$ has $2 n-1$ measure zero. Let $\tilde{\rho}$ be a smooth non-negative function vanishing precisely on $\sigma$, and let $M_{\epsilon}=\{z \in M: \tilde{\rho}>\epsilon\}$. If $f \in L_{\mathrm{loc}}^{1}(M)$ is $\mathrm{CR}$ on $M \backslash \sigma$ and satisfies the condition

$$
\int_{\partial M_{\epsilon} \cap K}|f(z)| d \lambda_{2 n-2}=o(1), \text { as } \epsilon \rightarrow 0,
$$

for all compacts $K$ in $\Omega$, then $f$ is a CR function (as stated in Definition 3.1). In fact this result can be extended to the case when $\rho$ is merely smooth. This may be used to construct examples of CR functions on quadratic cones. 
Lemma 3.1. (a) Let $M \subset \mathbb{C}^{n}$ be a real quadratic cone, and let $f \in L_{\text {loc }}^{1}(M)$ be $C R$ on $M^{\mathrm{reg}}$. If $f$ is bounded in a neighbourhood of $M^{\mathrm{sng}}$, then $f$ is $C R$ on $M$.

(b) Suppose further that $M^{\text {sng }}=\{0\}$ and

$$
f(z)=O\left(\frac{1}{|z|^{\alpha}}\right)
$$

where $\alpha<2 n-2$. Then $f$ is $C R$ on $M$.

Proof. (a) $M^{\operatorname{sing}}=\{\rho=0, d \rho=0\}$ is a real linear subspace of $\mathbb{C}^{n}$ of real dimension at most $2 n-2$. If $\operatorname{dim} M^{\operatorname{sing}}<2 n-2$ and $f$ is bounded, then (9) is clearly satisfied, and $f$ is $\mathrm{CR}$ on $M$. Suppose now that $\operatorname{dim} M^{\operatorname{sing}}=2 n-2$. After a suitable $\mathbb{R}$-linear change of variables in $\mathbb{C}^{n}=\mathbb{R}^{2 n}$ we may assume that $\{d \rho=0\}=\left\{x_{1}=x_{2}=0\right\}$. It is easy to see that after an additional change of variables, the defining function of $M$ admits the form $\rho=x_{1}^{2}-A x_{2}^{2}, A \in \mathbb{R}$. Thus $M$ reduces to the union of real hyperplanes, but this is ruled out by our assumption. This proves the assertion.

(b) We can take $\tilde{\rho}(z)=|z|^{2}$. Since $\lambda_{2 n-2}\left(\partial M_{\epsilon}\right)=O\left(\epsilon^{2 n-2}\right)$, equation (9) holds.

3.2. The jump formula and proof of Corollary 1.1. The jump formula (1], 8]) for CR functions generalizes the classical Sohockii-Plemelj formula for functions of one variable. If $\Omega$ is a domain in $\mathbb{C}^{n}$, with $H^{0,1}(\Omega)=0$ (e.g., $\Omega$ can be taken to be pseudoconvex), $M$ is a smooth hypersurface in $\Omega$ which divides $\Omega$ into two connected components $\Omega^{+}$and $\Omega^{-}$, and $f$ is a CR function on $M$, then there exist functions $F^{+} \in \mathcal{O}\left(\Omega^{+}\right)$and $F^{-} \in \mathcal{O}\left(\Omega^{-}\right)$such that the following holds:

$$
f=F^{+}-F^{-} .
$$

The latter equality is understood in an appropriate sense depending on the smoothness of $f$. In particular, if $f \in L_{\mathrm{loc}}^{1}$, then for any point $p \in M$, there exists a neighbourhood $U$ such that

$$
\lim _{\epsilon \rightarrow 0^{+}} \int_{M \cap U}\left|F^{+}(\zeta+\epsilon \nu(\zeta))-F^{-}(\zeta-\epsilon \nu(\zeta))-f(\zeta)\right| d \lambda_{2 n-1}(\zeta)=0,
$$

and $\nu(\zeta)$ is the unit normal vector to $M$ at $\zeta \in M$. And if $f$ is a Hölder continuous function on $M$ of class $\mathcal{C}^{\alpha}, 0<\alpha<1$, then the functions $F^{+}$and $F^{-}$extend to $M$ as $\mathcal{C}^{\alpha}$ functions.

Trépreau's theorem 21 now states that in the situation above, if $M$ is a $C^{2}$ smooth minimal hypersurface, then for any point $z \in M$ there exists a neighbourhood $U$ such that for any CR function $f$ on $M$ either $F^{+}$or $F^{-}$in (10) extends holomorphically to $U$. Thus any CR function admits a one-sided holomorphic extension.

We now consider an analog of the jump formula for real analytic hypersurfaces. If $\Omega$ is a domain in $\mathbb{C}^{n}$, with $H^{0,1}(\Omega)=0, M$ is a real analytic hypersurface in $\Omega$, and $f$ a CR function on $M$ then by Dolbeault's theorem the $\bar{\partial}$-closed current $f[M]^{0,1}$ of bidegree $(0,1)$ is $\bar{\partial}$-exact. Hence, there exists a current of degree zero, i.e., a distribution $F$ such that $\bar{\partial} F=f[M]^{0,1}$. Now $M$ divides $\Omega$ into two parts $\Omega^{ \pm}$as in Lemma 2.1 above. (We emphasize again that $\Omega^{+}$and $\Omega^{-}$need not be connected.) Since $f[M]^{0,1}$ has support on $M$, we have $\bar{\partial} F=0$ on $\Omega \backslash M$, and it follows that $F^{ \pm}:=\left.F\right|_{\Omega^{ \pm}} \in \mathcal{O}\left(\Omega^{ \pm}\right)$. As in $[8$, a Bochner-Martinelli type integral formula can be used to deduce the correspondence between the values of $F^{ \pm}$and $f$ at smooth points of $M$. This yields the jump formula (10) for CR functions defined 
on (singular) real analytic hypersurfaces. In this case, (10) holds only on $M^{\text {reg}}$, in a sense depending on the smoothness of $f$.

As an immediate consequence of the jump formula, we can deduce Corollary 1.1 from Theorem 1.1. Let $M$ be a quadratic cone in $\mathbb{C}^{n}$ which does not have two-sided support at the origin, and let $f$ be a CR function in a neighbourhood of 0 in $M$. By the jump formula, we write $f=F^{+}-F^{-}$, with $F^{ \pm} \in \mathcal{O}\left(\Omega^{ \pm}\right)$. By Theorem 1.1 one of the functions $F^{ \pm}$extends to a neighbourhood $U$ of 0 in $\mathbb{C}^{n}$. For definiteness assume that $F^{+}$extends to $\tilde{F}^{+} \in \mathcal{O}(U)$. Then we can take $F=\tilde{F}^{+}-F^{-}$. For the last statement of Corollary 1.1, note that by Lemma 3.1, the function $f$ is CR on $M$, and therefore, as above, $F=\tilde{F}^{+}-F^{-}$is the desired one-sided extension. The equality $\left.F\right|_{M^{\mathrm{reg}}}=f$ follows from [18. This completes the proof of the corollary.

Combining the jump formula with the results in [10] one may further conclude that if $M$ is a real analytic hypersurface that can be represented as the graph of a continuous function over a suitable domain in $\mathbb{C}^{n-1} \times \mathbb{R}$, then all $\mathrm{CR}$ functions on $M$ extend to one side of $M$, unless $M$ is non-minimal. However, as we saw in Section 2.2, a quadratic cone can never be represented as a graph of a continuous function. Therefore, Chirka's result cannot be used. In fact, the presence of a single non-smooth point on $M$ leads to new phenomena in the behaviour of CR functions, as illustrated in the introduction. Thus a different approach is needed to prove Theorem 1.1 .

\section{Facts from Linear Algebra.}

The following results in Linear Algebra will be used in the subsequent sections. We denote by $\operatorname{Sym}(2, \mathbb{R})$ the space of $2 \times 2$ real symmetric matrices, by $S L(2, \mathbb{R})$ (resp. $S L(2, \mathbb{C})$ ) the group of $2 \times 2$ real (resp. complex) matrices of determinant 1 , and by $S O(1,1)$ the group of $2 \times 2$ real matrices $g$ with $\operatorname{det} g=1$, such that $g^{t} A g=A$, where $A=\operatorname{diag}(1,-1)$ is the diagonal matrix with the diagonal entries 1 and -1 .

Lemma 4.1. (a) A matrix $k$ preserves the hermitian form $\operatorname{Im}\left(z_{1} \bar{z}_{2}\right)$ if and only if there exists $g \in S L(2, \mathbb{R})$ and $\theta \in \mathbb{R}$ such that $k=e^{i \theta} g$.

(b) If $P \in \operatorname{Sym}(2, \mathbb{R}), P \neq 0$, then there exists $g \in S L(2, \mathbb{R})$ such that $g^{t} P g$ has one of the following forms:

(i) $\pm \sqrt{\operatorname{det} P} I_{2}$, if $\operatorname{det} P>0$ (here $I_{2}$ is the $2 \times 2$ identity matrix);

(ii) $\pm \sqrt{-\operatorname{det} P} \operatorname{diag}(1,-1)$, if $\operatorname{det} P<0$;

(iii) $\operatorname{diag}(1,0)$, if $\operatorname{det} P=0$.

Proof. (a) Denote

$$
E=\left(\begin{array}{cc}
0 & 1 \\
-1 & 0
\end{array}\right)
$$

Then $\operatorname{Im}\left(z_{1} \overline{z_{2}}\right)=\frac{1}{2 i} \bar{z}^{t} E z$. Therefore, we have, $\bar{k}^{t}\left(\frac{1}{2 i} E\right) k=\frac{1}{2 i} E$, so that taking determinants, $|\operatorname{det} k|^{2}=1$. Consequently, we can write $k=e^{i \theta} g$, where $g \in S L(2, \mathbb{C})$, $\theta \in \mathbb{R}$. Then, $\bar{g}^{t} E g=E$, or $\bar{g}^{t} E=E g^{-1}$. From this it follows that $g \in S L(2, \mathbb{R})$. This completes the proof of part (a).

(b) There exists $g \in G L(2, \mathbb{R})$ such that

$$
g^{t} P g= \begin{cases} \pm I_{2}, & \text { if } \operatorname{det} P>0 \\ \pm \operatorname{diag}(1,-1), & \text { if } \operatorname{det} P<0\end{cases}
$$


If $\operatorname{det} g>0$, we can write $g=\delta h$, where $\delta \in \mathbb{R}$, and $h \in S L(2, \mathbb{R})$. Then $h^{t} P h= \pm \frac{1}{\delta^{2}} I_{2}$. If $\operatorname{det} g<0$, we can write $g=\delta J h$, where $\delta>0$,

$$
J=\left(\begin{array}{ll}
0 & 1 \\
1 & 0
\end{array}\right)
$$

and $h \in S L(2, \mathbb{R})$. In either case, we have

$$
h^{t} P h= \begin{cases} \pm \sqrt{\operatorname{det} P} I_{2}, & \text { if } \operatorname{det} P>0 \\ \pm \sqrt{-\operatorname{det} P} \operatorname{diag}(1,-1), & \text { if } \operatorname{det} P<0 .\end{cases}
$$

If $\operatorname{det} P=0$ but $P \neq 0$, then there is a $g \in G L(2, \mathbb{R})$ such that $g^{t} P g=\operatorname{diag}(1,0)$. We can write $g=\operatorname{diag}(1, \operatorname{det}(g)) h$, if $\operatorname{det} g>0$, and $g=\operatorname{diag}(1, \operatorname{det}(g)) J h$ if $\operatorname{det} g<0$, where $h \in S L(2, \mathbb{R})$, and $J$ is as in (12). Then $h^{t} P h=\operatorname{diag}(1,0)$ or $\operatorname{diag}(0,1)$.

Lemma 4.2. Let $Q \in \operatorname{Sym}(2, \mathbb{R})$, $\operatorname{det} Q \leq 0$ and the sum of the four entries of $Q$ is different from 0 . Then there is $k \in S O(1,1)$ such that $k^{t} Q k$ has at least one zero entry on the main diagonal.

Proof. Let $\mathbb{R}^{+}$be the group of positive reals under multiplication. Let $\sigma(\tau)=$ $\tau+\tau^{-1}, \delta(\tau)=\tau-\tau^{-1}$, and define

$$
\varphi(\tau)=\frac{1}{2}\left(\begin{array}{cc}
\sigma(\tau) & \delta(\tau) \\
\delta(\tau) & \sigma(\tau)
\end{array}\right)
$$

Then $\varphi: \mathbb{R}^{+} \rightarrow S O(1,1)$ is a group isomorphism. Assume that

$$
Q=\left(\begin{array}{cc}
p & q \\
q & r
\end{array}\right)
$$

and let $Q^{\prime}=\varphi(\tau)^{t} Q \varphi(\tau)$. Denoting the corresponding elements of $Q^{\prime}$ by primed letters, we compute

$$
\left\{\begin{array}{l}
p^{\prime}=\frac{1}{4}\left(\sigma^{2} p+2 \sigma \delta q+\delta^{2} r\right) \\
q^{\prime}=\frac{1}{4}\left(\sigma \delta p+\left(\sigma^{2}+\delta^{2}\right) q+\sigma \delta r\right) \\
r^{\prime}=\frac{1}{4}\left(\delta^{2} p+2 \sigma \delta q+\sigma^{2} r\right) .
\end{array}\right.
$$

We need to show that either $p^{\prime}(\tau)=0$ or $q^{\prime}(\tau)=0$ has a solution. After simplifications, we obtain

$$
\left\{\begin{array}{l}
(p+2 q+r) \tau^{2}+2(p-r) \tau^{2}+(p-2 q+r)=0 \\
(p+2 q+r) \tau^{2}-2(p-r) \tau^{2}+(p-2 q+r)=0 .
\end{array}\right.
$$

The possible solutions are contained in the formula:

$$
\tau^{2}=\frac{ \pm(p-r) \pm 2 \sqrt{q^{2}-p r}}{p+2 q+r} .
$$

By hypothesis the denominator is non-zero, and it is easy to see that at least one of the four values of the right hand side is a positive real number. 


\section{Normal form for quadratic cones in $\mathbb{C}^{2}$ : Proof of Theorem 1.2 .}

Recall that by Lemma 2.2, two quadratic cones which are biholomorphically equivalent are linearly equivalent, and thus cones with different hermitian signatures $(\pi, \nu), \pi \geq \nu$, are not biholomorphic and may be considered separately. The only possibilities are $(\pi, \nu)=(2,0),(1,1),(1,0)$ and $(0,0)$.

$(\pi, \nu)=(2,0)$. After a complex linear change of coordinates we may assume that the matrix $H$ in (6) is $I_{2}$. According to [20, Theorem II] there is a $2 \times 2$ unitary matrix $u$, such that $u^{t} S u=\operatorname{diag}(A, B)$, where $A, B$ are the singular values of the matrix $S$, i.e., the non-negative square roots of the eigenvalues of $\bar{S}^{t} S=\bar{S} S$. (This is a special case of "Singular Value Decomposition" of matrices.)

Therefore, there exists a linear change of variables such that in the new coordinates the defining equation of $M$ takes the form

$$
\rho(z)=\operatorname{Re}\left(A z_{1}^{2}+B z_{2}^{2}\right)+\left|z_{1}\right|^{2}+\left|z_{2}\right|^{2} .
$$

Permuting $z_{1}$ and $z_{2}$ if required, we have $0 \leq B \leq A$. Also, $A>1$, since $M$ must have dimension 3 .

If two cones of type $\mathcal{M}_{(2,0)}$ with parameters $(A, B)$ and $\left(A^{\prime}, B^{\prime}\right)$ are linearly equivalent, by [20, Theorem II] the diagonal matrices $\operatorname{diag}(A, B)$ and $\operatorname{diag}\left(A^{\prime}, B^{\prime}\right)$ must have the same singular values. Since $0 \leq B \leq A$ and $0 \leq B^{\prime} \leq A^{\prime}$, this means that $A=A^{\prime}$ and $B=B^{\prime}$. This proves the uniqueness of the normal form.

$(\pi, \nu)=(\mathbf{1}, \mathbf{1})$. Here, after a linear change of variables, we may assume that the defining equation of $M$ has the form

$$
\rho(z)=\operatorname{Re}\left(z^{t} S z\right)+\operatorname{Im}\left(z_{1} \bar{z}_{2}\right),
$$

where $S \in \operatorname{Sym}(2, \mathbb{C})$. If $\operatorname{det} S \neq 0$, we may further make a change of variables of the form $z^{*}=e^{i \theta} z$, so that $\operatorname{det} S>0$. (Such a change of variables preserves the hermitian part $\operatorname{Im}\left(z_{1} \overline{z_{2}}\right)$.) Let $S=P+i Q$, where $P=\left(p_{i j}\right)$ and $Q=\left(q_{i j}\right)$ are both in $\operatorname{Sym}(2, \mathbb{R})$. Then, a computation shows that $\operatorname{det} S=\operatorname{det} P-\operatorname{det} Q+i\left(q_{11} p_{22}+\right.$ $\left.p_{11} q_{22}-2 q_{12} p_{12}\right)$. Since $\operatorname{det} S \geq 0$,

$$
\operatorname{det} S=\operatorname{det} P-\operatorname{det} Q,
$$

and

$$
q_{11} p_{22}+p_{11} q_{22}-2 q_{12} p_{12}=0 .
$$

We consider three cases depending on the sign of $\operatorname{det} P$.

Case 1. Suppose that $\operatorname{det} P>0$. By Lemma 4.1(b) there exists $g \in S L(2, \mathbb{R})$ such that $g^{t} P g= \pm \sqrt{\operatorname{det} P} I_{2}$. Since $g^{t} Q g \in \operatorname{Sym}(2, \mathbb{R})$, there is $k \in S O(2, \mathbb{R}) \subset$ $S L(2, \mathbb{R})$ such that $k^{t}\left(g^{t} Q g\right) k=\operatorname{diag}\left(\lambda_{1}, \lambda_{2}\right)$. Since $g k \in S L(2, R)$, by Lemma 4.1 the change of variables $z=g k z^{*}$ leaves $\operatorname{Im}\left(z_{1} \overline{z_{2}}\right)$ invariant, and in the new coordinates we have

$$
\rho\left(z^{*}\right)=\operatorname{Re}\left(z^{* t} S^{\prime} z^{*}\right)+\operatorname{Im}\left(z_{1}^{*} \bar{z}_{2}^{*}\right),
$$

where $S^{\prime}= \pm \sqrt{\operatorname{det} P} I_{2}+i \operatorname{diag}\left(\lambda_{1}, \lambda_{2}\right)$. It follows from (17) that $\pm \sqrt{\operatorname{det} P}\left(\lambda_{1}+\right.$ $\left.\lambda_{2}\right)=0$. Consequently, $\lambda_{1}=-\lambda_{2}=\sqrt{-\operatorname{det} Q}$, and therefore, $S^{\prime}=\operatorname{diag}(A, \bar{A})$, where $A=\sqrt{\operatorname{det} P}+i \sqrt{-\operatorname{det} Q}$. Note that $A \neq 0$, and $\operatorname{Re} A, \operatorname{Im} A \geq 0$. Hence, $M$ is equivalent to a cone of type $\mathcal{M}_{(1,1)}^{2}$.

Case 2. Suppose that $\operatorname{det} P<0$. Then by Lemma 4.1(b) there exists $g \in$ $S L(2, \mathbb{R})$ such that $g^{t} P g$ is the matrix of the form $\pm \sqrt{-\operatorname{det} P} \operatorname{diag}(1,-1)$. Since $\operatorname{det} Q=\operatorname{det} P-\operatorname{det} S \leq 0$, and thanks to (16) and (17) above, it follows that the 
sum of the entries of $g^{t} \mathrm{Pg}$ cannot be zero, so by Lemma 4.2 there exists a matrix $k \in S O(1,1)$ such that for $Q^{\prime}=k^{t}\left(g^{t} Q g\right) k=\left(q_{i j}^{\prime}\right)$, where either $q_{11}^{\prime}$ or $q_{22}^{\prime}$ vanishes. Since the subgroup $S O(1,1) \subset S L(2, \mathbb{R})$ preserves $P^{\prime}=g^{t} P g$, it follows from (17) that either $q_{11}^{\prime} \sqrt{-\operatorname{det} P}=0$, or $q_{22}^{\prime} \sqrt{-\operatorname{det} P}=0$. In either case we deduce that $q_{11}^{\prime}=q_{22}^{\prime}=0$. After the change of variables $z=k g z^{*}$ the defining equation of $M$ has the form (18), with

$$
S^{\prime}=\left(\begin{array}{cc}
a & i \mu \\
i \mu & -a
\end{array}\right)
$$

where $a= \pm \sqrt{-\operatorname{det} P}$, and $\mu= \pm \sqrt{-\operatorname{det} Q}$. Since $\operatorname{det} S=\operatorname{det} P-\operatorname{det} Q \geq 0$, it follows that $|\mu| \geq|a|$. After an additional change of variables

$$
\left\{\begin{array}{ccc}
z_{1}^{*} & =z_{1}+i z_{2} \\
z_{2}^{*} & = & -\left(i z_{1}+z_{2}\right)
\end{array}\right.
$$

in the $\left(z_{1}, z_{2}\right)$ coordinates, the equation of $M$ becomes:

$$
\begin{gathered}
\operatorname{Re}\left[a\left(z_{1}+i z_{2}\right)^{2}-2 i \mu\left(z_{1}+i z_{2}\right)\left(i z_{1}+z_{2}\right)-a\left(i z_{1}+z_{2}\right)^{2}\right] \\
+\operatorname{Im}\left(\left(z_{1}+i z_{2}\right)\left(i \bar{z}_{1}-\bar{z}_{2}\right)\right) \\
=2 \operatorname{Re}\left[(a+\mu) z_{1}^{2}+(\mu-a) z_{2}^{2}\right]+\left|z_{1}\right|^{2}-\left|z_{2}\right|^{2} .
\end{gathered}
$$

We make a further linear change of variables $z_{1}=\varepsilon_{1} z_{1}^{*}, z_{2}=\varepsilon_{2} z_{2}^{*}$, where the $\varepsilon_{j}$ are $i$ or 1 . It is clear that the $\varepsilon_{j}$ can be so chosen that $M$ is given by

$$
\operatorname{Re}\left(A z_{1}^{2}+B z_{2}^{2}\right)+\left|z_{1}\right|^{2}-\left|z_{2}\right|^{2}=0
$$

with $A=2|a+\mu|, B=2|\mu-a|$. Interchanging $z_{1}$ and $z_{2}$ if required, we get $\mathcal{M}_{(1,1)}^{1}$.

Case 3. Now let $\operatorname{det} P=0$, and let the sum of entries of $g^{t} P g$ be nonzero. First suppose that $\operatorname{det} Q<0$. Then there exists $g \in S L(2, \mathbb{R})$ such that $g^{t} Q g=$ $\pm \sqrt{-\operatorname{det} Q} \operatorname{diag}(1,-1)$. By Lemma 4.2 there exists $k \in S O(1,1)$ such that $k^{t}\left(g^{t} P g\right) k$ becomes

$$
\left(\begin{array}{cc}
\alpha^{\prime} & 0 \\
0 & 0
\end{array}\right) \text { or }\left(\begin{array}{cc}
0 & 0 \\
0 & \gamma^{\prime}
\end{array}\right)
$$

Note that the non-diagonal entries vanish since $\operatorname{det} P=0$. From this and (17) we deduce that $P=0$. It follows then that $M$ is equivalent to a cone of the form (with $\mu \in \mathbb{R})$

$$
\operatorname{Re}\left(i \mu\left(z_{1}^{2}-z_{2}^{2}\right)\right)+\operatorname{Im}\left(z_{1} \overline{z_{2}}\right)=0 .
$$

We again make the change of variables (20). In the $\left(z_{1}, z_{2}\right)$ coordinates, the cone is given by

$$
2 \operatorname{Re}\left[i \mu\left(z_{1}^{2}-z_{2}^{2}\right)\right]+\left|z_{1}\right|^{2}-\left|z_{2}\right|^{2}=0
$$

which after another linear change of coordinates reduces to form $\mathcal{M}_{(1,1)}^{1}$ with $A=$ $B=|\mu|$.

Now suppose that $\operatorname{det} Q=0$. If $P \neq 0$, then there exists a $g \in S L(2, \mathbb{R})$ such that $g^{t} P g=\operatorname{diag}(1,0)$. Let $Q^{\prime}=g^{t} Q g=\left(q_{i j}^{\prime}\right)$. Then from (17) we conclude that $q_{22}^{\prime}=0$. Since $\operatorname{det} Q^{\prime}=0, q_{12}^{\prime}=0$, and $Q^{\prime}=\operatorname{diag}\left(q_{11}^{\prime}, 0\right)$. Thus we may assume that the defining equation of $M$ has the form

$$
\rho(z)=\operatorname{Re}\left(a z_{1}^{2}\right)+\operatorname{Im} z_{1} \bar{z}_{2}=0
$$

where $a=1+i q_{11}^{\prime}$. Let $\alpha$ be a square root of $a$. After a change of variables $z_{1}=\alpha z_{1}^{*}, z_{2}=\frac{1}{\alpha} z_{2}^{*}$, we arrive at $\mathcal{M}_{(1,1)}^{3}$. If $P=0, Q \neq 0$, a similar argument 
shows that $M$ can be given by (23) with $a \neq 0$, and again we get $\mathcal{M}_{(1,1)}^{3}$. Finally, $P=Q=0$ corresponds to $\mathcal{M}_{(1,1)}^{1}$ with $A=B=0$.

Case 4. After reducing the matrix $Q$ to the diagonal form $q \operatorname{diag}(1,-1), q \in \mathbb{R} \backslash$ $\{0\}$, assume that in the new coordinates the entries of $P$ satisfy $p_{11}+p_{22}+2 p_{12}=0$. It then follows from (17) that

$$
P=p\left(\begin{array}{cc}
1 & -1 \\
-1 & 1
\end{array}\right)
$$

where $p \in \mathbb{R}$. Thus the equation of the cone becomes

$$
\operatorname{Re}\left((p+i q) z_{1}^{2}-2 p z_{1} z_{2}+(p-i q) z_{2}^{2}\right)+\operatorname{Im}\left(z_{1} \overline{z_{2}}\right)=0 .
$$

If $p=0$, this reduces to the form considered in Case 3 , i.e., $\mathcal{M}_{(1,1)}^{1}$. If $q<0$, we make the coordinate change $z^{*}=i z$. If now $p<0$, we make the additional change of coordinates $z_{1}^{*}=z_{2}, z_{2}^{*}=z_{1}$, so that we have $p>0, q>0$. Finally, we make the coordinate change $z_{1}^{*}=\sqrt{p}\left(z_{1}-z_{2}\right), z_{2}^{*}=\frac{1}{2 \sqrt{p}}\left(z_{1}+z_{2}\right)$, which reduces the cone to type $\mathcal{M}_{(1,1)}^{4}$, with $A=2 q$.

We now consider uniqueness of the normal forms of hermitian signature $(1,1)$. Let $M_{1}, M_{2}$ be biholomorphic cones with $(\pi, \nu)=(1,1)$, such that each is given in one of the normal forms of Theorem 1.2. If the harmonic part of $M_{j}$ is $\operatorname{Re}\left(z^{t} S_{j} z\right)$, then it is easy to see that rank of the matrices $S_{1}$ and $S_{2}$ are equal, in particular, either (1) for $j=1,2, \operatorname{det} S_{j} \neq 0$ or (2) $\operatorname{det} S_{1}=0=\operatorname{det} S_{2}$. We consider case (1) first.

Lemma 5.1. Suppose that the same quadratic cone $M$ is represented in two linear coordinate systems by:

$$
\operatorname{Re}\left(z^{t} S_{1} z\right)+\operatorname{Im}\left(z_{1} \overline{z_{2}}\right)=0
$$

and

$$
\operatorname{Re}\left(z^{t} S_{2} z\right)+\operatorname{Im}\left(z_{1} \overline{z_{2}}\right)=0,
$$

where each $S_{j} \in \operatorname{Sym}(2, \mathbb{C})$ is such that $\operatorname{det} S_{j}>0$. Then

(i) $\operatorname{det} S_{1}=\operatorname{det} S_{2}$, and

(ii) If $S_{j}=P_{j}+i Q_{j}, P_{j}, Q_{j} \in \operatorname{Sym}(2, \mathbb{R})$, then $\operatorname{det} P_{1}=\operatorname{det} P_{2}$ and $\operatorname{det} Q_{1}=$ $\operatorname{det} Q_{2}$.

Proof. Let $k \in G L(2, \mathbb{C})$ be the matrix defining the change of variables between the coordinates in which equations (24) and (25) represent the same cone $M$. Since the hermitian part is invariant under a linear change of coordinates, by Lemma 4.1(a) $k=e^{i \theta} g$, where $g \in S L(2, \mathbb{R})$, and $\theta \in \mathbb{R}$. Therefore, $S_{2}=e^{2 i \theta}\left(g^{t} S_{1} g\right)$, so that $\operatorname{det} S_{2}=e^{4 i \theta} \operatorname{det} S_{1}$. By the positivity of $\operatorname{det} S_{j}$ we have $e^{4 i \theta}=1$ and $\operatorname{det} S_{2}=$ $\operatorname{det} S_{1}$.

We see that $\epsilon=e^{i \theta}$ is a fourth root of unity. Therefore,

$$
\begin{aligned}
P_{2}+i Q_{2} & =k^{t}\left(P_{1}+i Q_{1}\right) k \\
& =\epsilon^{2} g^{t}\left(P_{1}+i Q_{1}\right) g \\
& = \pm\left(g^{t} P_{1} g+i g^{t} Q_{1} g\right) .
\end{aligned}
$$

Since the entries of $g \in S L(2, \mathbb{R})$ are real, it follows that $P_{2}= \pm g^{t} P_{1} g$ and $Q_{2}=$ $\pm g^{t} Q_{1} g$, and on taking determinants, we have $\operatorname{det} P_{2}=\operatorname{det} P_{1}$ and $\operatorname{det} Q_{2}=\operatorname{det} Q_{1}$. 
First suppose that both $M_{1}$ and $M_{2}$ are of type $\mathcal{M}_{(1,1)}^{2}$, and are given by (24) and (25) respectively, where $S_{j}=\operatorname{diag}\left(A_{j}, \overline{A_{j}}\right)$ with $\operatorname{Re} A_{j}>0$ and $\operatorname{Im} A_{j} \geq 0$. By Lemma 5.1, $\operatorname{det} P_{1}=\left(\operatorname{Re} A_{1}\right)^{2}=\left(\operatorname{Re} A_{2}\right)^{2}=\operatorname{det} P_{2}$, and $\operatorname{det} Q_{1}=-\left(\operatorname{Im} A_{1}\right)^{2}=$ $-\left(\operatorname{Im} A_{2}\right)^{2}=\operatorname{det} Q_{2}$. Therefore, $A_{1}=A_{2}$, and $M_{1}$ and $M_{2}$ have the same normal form.

Now suppose that $M_{1}$ is of type $\mathcal{M}_{(1,1)}^{1}$ and given by $\operatorname{Re}\left(a z_{1}^{2}+b z_{2}^{2}\right)+\left|z_{1}\right|^{2}-\left|z_{2}\right|^{2}=$ 0 with $0<a \leq b$. We make the change of variables

$$
\begin{aligned}
& z_{1}^{*}=\frac{1}{2}\left(z_{1}+i z_{2}\right) \\
& z_{2}^{*}=\frac{1}{2 i}\left(z_{1}-i z_{2}\right),
\end{aligned}
$$

so that the equation of $M_{1}$ takes the form (15) with

$$
S=\left(\begin{array}{cc}
a-b & i(a+b) \\
i(a+b) & b-a
\end{array}\right) \text {. }
$$

Therefore, $\operatorname{det} S=4 a b>0$. Writing $S=P+i Q$, we have $\operatorname{det} P=-(b-a)^{2}$ and $\operatorname{det} Q=-(a+b)^{2}$. If $M_{2}$ is of type $\mathcal{M}_{(1,1)}^{2}$, then $S_{2}=P_{2}+i Q_{2}=\operatorname{diag}(A, \bar{A})$ with $\operatorname{det} P_{2}=(\operatorname{Re} A)^{2}$ and $\operatorname{det} Q_{2}=-(\operatorname{Im} A)^{2}$. Since $\operatorname{Re} A>0$, it follows from Lemma 5.1 that $M_{2}$ is not biholomorphic to $M_{1}$. Therefore, $M_{2}$ is also of type $\mathcal{M}_{(1,1)}^{1}$. Assume that $M_{2}$ is given by $\operatorname{Re}\left(a^{\prime} z_{1}^{2}+b^{\prime} z_{2}^{2}\right)+\left|z_{1}\right|^{2}-\left|z_{2}\right|^{2}=0$, with $0 \leq a^{\prime} \leq b^{\prime}$. Then $-\left(b^{\prime}-a^{\prime}\right)^{2}=-(b-a)^{2}$ and $-\left(a^{\prime}+b^{\prime}\right)^{2}=-(a+b)^{2}$, hence, $a^{\prime}=a$ and $b^{\prime}=b$.

For case (2) suppose that $M_{j}, j=1,2$ are cones of hermitian signature $(1,1)$ in normal form with harmonic parts $\operatorname{Re}\left(z^{t} S_{j} z\right)$, where $\operatorname{det} S_{1}=0=\operatorname{det} S_{2}$. By inspection of the table of normal forms, $M_{j}$ are of type $\mathcal{M}_{(1,1)}^{3}$, or of type $\mathcal{M}_{(1,1)}^{1}$, with $B=0$. In the latter case we can write the equation of the cone as

$$
\operatorname{Re}\left(a z^{t} E z\right)+\operatorname{Im}\left(z_{1} \overline{z_{2}}\right)=0
$$

where $a=\frac{1}{2} A$, and

$$
E=\left(\begin{array}{cc}
1 & i \\
i & -1
\end{array}\right)
$$

Note that no cone of type $\mathcal{M}_{(1,1)}^{1}$ is linearly equivalent to the cone $\mathcal{M}_{(1,1)}^{3}$. Indeed, if not, then there would exist $g \in S L(2, \mathbb{R}), \theta \in \mathbb{R}$ and $a \geq 0$ such that $e^{2 i \theta} g^{t} \operatorname{diag}(1,0) g=a E$. Let $e^{2 i \theta} g^{t} \operatorname{diag}(1,0) g=\left(p_{j k}\right)$, then $\frac{p_{11}}{p_{22}}$ is a positive real number. However, on the right hand side, the ratio of the diagonal entries is -1 , which is a contradiction.

Now we show that if $a \neq a^{\prime}$, the cones (26) and

$$
\operatorname{Re}\left(a^{\prime} z^{t} E z\right)+\operatorname{Im}\left(z_{1} \overline{z_{2}}\right)=0
$$

are not linearly equivalent. If they were equivalent, there would exist $g \in S L(2, \mathbb{R})$ and $\theta \in \mathbb{R}$ such that $e^{2 i \theta} g^{t}(a E) g=a^{\prime} E$. Assuming without loss of generality that $a \neq 0$, we have (with $\mu=\frac{a^{\prime}}{a} e^{-2 i \theta}$ )

$$
g^{t} E=\mu E g^{-1} .
$$

From this we easily conclude that $g$ is the identity, and therefore, $\mu=1$, and $a=a^{\prime}$.

The uniqueness of cones of type $\mathcal{M}_{(1,1)}^{4}$ follows the same way as for types $\mathcal{M}_{(1,1)}^{1}$ and $\mathcal{M}_{(1,1)}^{2}$, using Lemma 5.1. The uniqueness of normal forms with hermitian signature $(1,1)$ follows. 
For later use we note the following.

Corollary 5.1. For the cone $M=\{\rho=0\}$, where $\rho$ is as in (15), assume that $\operatorname{det} S \geq \frac{1}{4}$, and $\operatorname{det} P<0$. Then $M$ is of type $\mathcal{M}_{(1,1)}^{1}$ with $A \neq B$ and $A \geq 1$.

Proof. It was shown in Case 2 above that such cones can be reduced to type $\mathcal{M}_{(1,1)}^{1}$ with $A \neq B$. Using the same notation, we have

$$
\begin{aligned}
A B & =4\left|\mu^{2}-a^{2}\right| \\
& =4\left|\operatorname{det} S^{\prime}\right|, \text { where } S^{\prime} \text { is as in equation (19) } \\
& =4|\operatorname{det} S|, \text { by Lemma } 5.1(\mathrm{i}) \\
& \geq 1
\end{aligned}
$$

Thus at least one of $A$ and $B$ must be greater than or equal to 1 . Since $A \geq B$, it follows that $A \geq 1$.

$(\boldsymbol{\pi}, \boldsymbol{\nu})=(\mathbf{1}, \mathbf{0})$. Such a cone is given by $\rho=0$, where $\rho\left(z_{1}, z_{2}\right)=\operatorname{Re}\left[A z_{1}^{2}+\right.$ $\left.2 B z_{1} z_{2}+C z_{2}^{2}\right]+\left|z_{1}\right|^{2}$. If $C \neq 0$, then

$$
A z_{1}^{2}+2 B z_{1} z_{2}+C z_{2}^{2}=\left(A-\frac{B^{2}}{C}\right) z_{1}^{2}+C\left(z_{2}+\frac{B}{C} z_{1}\right)^{2} .
$$

Therefore, if we set $w_{1}=e^{i \theta_{1}} z_{1}$ and $w_{2}=e^{i \theta_{2}} \sqrt{C}\left(z_{2}+\frac{B}{C} z_{1}\right)$, we obtain form $\mathcal{M}_{(1,0)}^{1}$ for an appropriate choice of $\theta_{1}$ and $\theta_{2}$. If $C=0$ and $B \neq 0$, we make the change of variables

$$
\begin{aligned}
& z_{1}^{*}=z_{1} \\
& z_{2}^{*}=2 B z_{2}+A z_{1},
\end{aligned}
$$

thus reducing the cone to type $\mathcal{M}_{(1,0)}^{2}$. If $C=B=0$, the defining function is of the form $\operatorname{Re}\left(A z_{1}^{2}\right)+\left|z_{1}\right|^{2}$. But then $\operatorname{dim} M<3$ if $|A| \leq 1$, and $M$ is reducible if $|A|>1$, which violates the assumption.

We now show that the normal forms are unique. If $M$ is non-minimal, it cannot be of type $\mathcal{M}_{(1,0)}^{1}$. Suppose that the cone $\operatorname{Re}\left(A z_{1}^{2}+z_{2}^{2}\right)+\left|z_{1}\right|^{2}=0$ is biholomorphic to the cone $\operatorname{Re}\left(A^{\prime} z_{1}^{2}+z_{2}^{2}\right)+\left|z_{1}\right|^{2}=0$. Then the linear biholomorphism between the two cones must map the hermitian form $\left|z_{1}\right|^{2}$ to itself, and thus must be of the form

$$
\begin{aligned}
& z_{1}^{*}=e^{i \theta} z_{1} \\
& z_{2}^{*}=p z_{1}+q z_{2} .
\end{aligned}
$$

It follows that $A^{\prime}=A$.

$(\boldsymbol{\pi}, \boldsymbol{\nu})=(\mathbf{0}, \mathbf{0})$. Writing the defining function as $\operatorname{Re}(q)=0$, where $q$ is a complex quadratic form, we can diagonalize $q$ by a change of coordinates to obtain $q=z_{1}^{2}$ or $q=z_{1}^{2}+z_{2}^{2}$, depending on the rank of $q$. However, $\operatorname{Re}\left(z_{1}^{2}\right)=x_{1}^{2}-y_{1}^{2}$ is reducible, and we are left with type $\mathcal{M}_{(0,0)}^{1}$. 


\section{Extension from QuAdratic COnes: Proof of TheOrem 1.1}

If (b) holds, then $M$ does not have two-sided support at 0 . Indeed, if not, then there exist complex analytic hypersurfaces $A^{ \pm}=\left\{\varphi^{ \pm}=0\right\} \subset \overline{\Omega^{ \pm}}$. After shrinking $\Omega$ if necessary, the function $1 / \varphi^{ \pm}$is holomorphic in $\Omega^{\mp}$ and does not extend to the origin. This proves $(\mathrm{b}) \Rightarrow(\mathrm{a})$.

The proof of the other direction consists of two steps. First we will consider the case $n=2$. In the next step, we will consider cones in $\mathbb{C}^{n}, n>2$, and reduce it to the $n=2$ case by a slicing argument. In both cases the extension will be derived from the Kontinuitätssatz. We note here that in all cases the constructed family of analytic discs is not contained in $\Omega^{-}$when $\Omega^{-}$consists of two components.

6.1. (a) $\Rightarrow(\mathbf{b})$ for $\boldsymbol{n}=\mathbf{2}$. After a linear change of coordinates, we may assume that $M=\{\rho=0\}$ is in the normal form of Theorem 1.2. After rescaling the coordinates, we may further assume that $\Omega=\left\{z \in \mathbb{C}^{n}:|z|<2\right\}$. Let $\mathbb{B}^{2}$ denote the unit ball in $\mathbb{C}^{2}$.

$\mathcal{M}_{(\mathbf{2}, \mathbf{0})}$. Suppose $M=\left\{\operatorname{Re}\left(A z_{1}^{2}+B z_{2}^{2}\right)+\left|z_{1}\right|^{2}+\left|z_{2}\right|^{2}=0\right\}$. Consider

$$
D_{\epsilon}=\left\{z \in \mathbb{B}^{2}: A z_{1}^{2}+B z_{2}^{2}=\epsilon\right\} .
$$

Let $\left(z_{1}, z_{2}\right) \in D_{\epsilon}$, with $\epsilon \geq 0$, then

$$
\rho\left(z_{1}, z_{2}\right)=\epsilon+\frac{1}{A}\left|\epsilon-B z_{2}^{2}\right|+\left|z_{2}\right|^{2} \geq 0
$$

with equality iff $z_{1}=z_{2}=\epsilon=0$. Thus, $D_{0} \cap M=\{0\}$ and for $\epsilon>0$, we have $D_{\epsilon} \subset \Omega^{+}$. Thus any $F^{+} \in \mathcal{O}\left(\Omega^{+}\right)$extends to a neighbourhood of 0 .

$\mathcal{M}_{(\mathbf{1}, \mathbf{1})}^{\mathbf{1}}$. We consider several cases:

(1) $B \leq A \leq 1$. In this case, $M$ has two-sided support at 0 , since $\left.\rho\right|_{\left\{z_{2}=0\right\}} \geq 0$ and $\left.\rho\right|_{\left\{z_{1}=0\right\}} \leq 0$.

(2) $B<1 \leq A$. In this case we let $D_{\epsilon}=\left\{\left(z_{1}, z_{2}\right) \in \mathbb{B}^{2}: z_{1}=i \epsilon\right\}$. Then $M \cap D_{0}=\{0\}$, and if $\epsilon>0$, we have

$$
\rho\left(i \epsilon, z_{2}\right)=\operatorname{Re}\left(B z_{2}^{2}\right)+\epsilon^{2}(1-A)-\left|z_{2}\right|^{2}<0,
$$

which shows that $D_{\epsilon} \subset \Omega^{-}$.

(3) $1 \leq B \leq A$. If $A=B$, then $M$ is clearly non-minimal. Therefore we assume that $B<A$.

$$
D_{\epsilon}=\left\{z \in \mathbb{B}^{2}: A z_{1}^{2}+B z_{2}^{2}=\epsilon\right\} .
$$

Let $\epsilon \geq 0$, and $\left(z_{1}, z_{2}\right) \in D_{\epsilon}$. Then, $z_{1}^{2}=\frac{1}{A}\left(\epsilon-B z_{2}^{2}\right)$, so that

$$
\rho\left(z_{1}, z_{2}\right)=\epsilon+\frac{1}{A}\left|\epsilon-B z_{2}^{2}\right|-\left|z_{1}\right|^{2} .
$$

Now,

$$
\begin{aligned}
\frac{1}{A^{2}}\left|\epsilon-B z_{2}^{2}\right|^{2}-\left(\epsilon-\left|z_{2}\right|^{2}\right)^{2} & =-\left(1-\frac{1}{A^{2}}\right) \epsilon^{2}-\left(1-\frac{B^{2}}{A^{2}}\right)\left|z_{2}\right|^{4} \\
& -2 \epsilon\left[\left(1-\frac{B}{A^{2}}\right) x_{2}^{2}+\left(1+\frac{B}{A^{2}}\right) y_{2}^{2}\right] \\
& \leq 0
\end{aligned}
$$

with strict inequality unless $\epsilon=z_{1}=z_{2}=0$. So $D_{0} \cap M=\{0\}$, and $D_{\epsilon} \subset \Omega^{-}$if $\epsilon>0$. 
For later use we note the following consequence of the above arguments and Corollary 5.1 .

Corollary 6.1. Let $M$ be a cone defined by $\{\rho=0\}$, where $\rho$ is as in equation (15), and $\operatorname{det} S \geq \frac{1}{4}$. Let $S=P+i Q$, with $P, Q$ real. If $\operatorname{det} P<0$, then either every function in $\mathcal{O}\left(\Omega^{+}\right)$or in $\mathcal{O}\left(\Omega^{-}\right)$extends to a neighbourhood of the origin.

$\mathcal{M}_{(1,1)}^{2}$. Let $\lambda_{1}$ be a real number such that

$$
e^{2 i \lambda_{1}}=-\frac{A}{\bar{A}}
$$

and let $\lambda_{2}=\lambda_{1}+\pi$. Note that $\lambda_{j}$ are not integer multiples of $\pi$. Consider the complex lines $\Lambda_{j}=\left\{z \in \mathbb{C}^{2}: z_{2}=e^{i \lambda_{j}} z_{1}\right\}$ for $j=1,2$. Then

$$
\rho\left(z_{1}, e^{i \lambda_{j}} z_{1}\right)=\operatorname{Re}\left[A z_{1}^{2}+\bar{A} e^{2 i \lambda_{j}} z_{1}^{2}\right]+\operatorname{Im}\left(z_{1} \cdot e^{-i \lambda_{j}} \overline{z_{1}}\right)=-\left|z_{1}\right|^{2} \sin \lambda_{j} .
$$

Since $\sin \lambda_{2}=\sin \left(\lambda_{1}+\pi\right)=-\sin \lambda_{1}$, it follows that $\Lambda_{j}$ lie in two different sets of $\Omega^{ \pm}$. Therefore, $M$ admits two-sided support.

$\mathcal{M}_{(1,0)}^{1}$. We have

$$
\rho(z)=\operatorname{Re}\left(A z_{1}^{2}+z_{2}^{2}\right)+\left|z_{1}\right|^{2}=(1+A) x_{1}^{2}+(1-A) y_{1}^{2}+x_{2}^{2}-y_{2}^{2} .
$$

Let $D_{\epsilon}=\left\{z \in \mathbb{B}^{2}: A z_{1}^{2}+z_{2}^{2}=\epsilon\right\}$. If $\epsilon>0$, for $z \in D_{\epsilon}$, we have $\rho(z)=\epsilon+\left|z_{1}\right|^{2} \geq 0$, with strict inequality unless $\epsilon=z_{1}=z_{2}=0$. Therefore, $D_{0} \cap M=\{0\}$, and $D_{\epsilon} \subset \Omega^{+}$, if $\epsilon>0$.

$\mathcal{M}_{(\mathbf{1}, \mathbf{0})}^{2}, \mathcal{M}_{(\mathbf{0}, \mathbf{0})}^{1}$. For these types, $M$ is non-minimal.

6.2. (a) $\Rightarrow$ (b) for $n>2$. We first note that if $M \subset \mathbb{C}^{n}, n \geq 3$, is a quadratic cone, and $L$ is a two dimensional complex linear subspace of $\mathbb{C}^{n}$ such that $M^{\prime}=M \cap L$ (viewed as a cone in $L$ ) does not have two-sided support, then all functions from one side of $M$ extend to a neighbourhood of the origin. Therefore, for $M$, which does not have two-sided support, it suffices to construct a two dimensional subspace $L \subset \mathbb{C}^{n}$ so that $M \cap L$ does not have two-sided support. We assume that $M$ is given by (6) and consider different hermitian signatures $(\pi, \nu), \pi \geq \nu$.

$\boldsymbol{\pi} \geq \mathbf{2}$. Without loss of generality,

$$
\bar{z}^{t} H z=\left|z_{1}\right|^{2}+\left|z_{2}\right|^{2}+\sum_{j=3}^{n} \varepsilon_{j}\left|z_{j}\right|^{2}, \quad \varepsilon_{j} \in\{-1,0,1\},
$$

and

$$
z^{t} S z=\operatorname{Re}\left(A z_{1}^{2}+B z_{2}^{2}+a z_{2} z_{3}+b z_{3}^{2}+c z_{1} z_{3}+s(z)\right),
$$

where $A, B \geq 0, a, b, c \in \mathbb{C}$, and $s(z)$ does not contain monomials depending on $z_{1}, z_{2}$ and $z_{3}$ only. We consider three possibilities below.

(i) Either $A>1$ or $B>1$. Let

$$
L=\left\{z \in \mathbb{C}^{n}: z_{j}=0, j=3,4, \ldots, n\right\}
$$

be the two-dimensional linear subspace of $\mathbb{C}^{n}$, and let $M^{\prime}=M \cap L$. Then as in the case $\mathcal{M}_{(2,0)}$ above, we obtain a sequence of analytic discs $\left\{D_{\epsilon}\right\}_{\epsilon>0}$ in $L \backslash M^{\prime} \subset \mathbb{C}^{n} \backslash M$ with the limit disc $D_{0}$ touching $M^{\prime}$ (and therefore $M$ ) at the origin.

(ii) If $A, B \leq 1$, and $A B<1$, we consider $L$ as in (31). Then the set $M^{\prime}=$ $M \cap L$ has dimension at most one. Clearly, there exists a sequence of analytic discs $\left\{D_{\epsilon}\right\}_{\epsilon \geq 0} \subset L$, such that $D_{\epsilon} \cap M^{\prime}=\varnothing$, for $\epsilon>0$, and $D_{0} \cap M^{\prime}=\{0\}$. 
(iii) Suppose now $A=B=1$, then $\operatorname{dim} M^{\prime}=2$. In this case we slightly change the subspace $L$. Note that the defining function of $M$ cannot depend on $z_{1}$ and $z_{2}$ only, since otherwise, $\operatorname{dim} M<2 n-1$. Assume first that not all $\varepsilon_{j}$ in (29) equal zero, say, $\varepsilon_{3} \neq 0$. If in (30), $a \neq 0$, we consider

$$
L=\left\{z \in \mathbb{C}^{n}: z_{4}=\cdots=z_{n}=0, z_{3}=\alpha z_{2}\right\}
$$

with $\alpha>0$. Then

$$
M \cap L=\left\{\operatorname{Re}\left(z_{1}^{2}+\left(1+\alpha a+\alpha^{2} b\right) z_{2}^{2}+c z_{1} z_{2}\right)+\left|z_{1}\right|^{2}+\left(1+\varepsilon_{3} \alpha^{2}\right)\left|z_{2}\right|^{2}=0\right\} .
$$

We claim that for any choice of $a, b$ and $c$, there exist $\alpha>0$, arbitrarily close to zero, and a linear change of variables in $\left(z_{1}, z_{2}\right)$ in which the equation of $M \cap L$ becomes

$$
\operatorname{Re}\left(A^{\prime} z_{1}^{2}+B^{\prime} z_{2}^{2}\right)+\left|z_{1}\right|^{2}+\left|z_{2}\right|^{2}=0
$$

and either $A^{\prime}$ or $B^{\prime}$ is different from 1 (then our result will follow from parts (i) and (ii) above.) Indeed, since the hermitian part is positive definite, the harmonic part is always diagonalizable. Therefore, we only need to verify that $A^{\prime}$ and $B^{\prime}$ are not both 1 . After a linear change of variables, (33) can be written in the form

$$
\operatorname{Re}\left(z^{t} S^{\prime} z\right)+\left|z_{1}\right|^{2}+\left|z_{2}\right|^{2}=0
$$

where

$$
S^{\prime}=\left(\begin{array}{cc}
1 & \frac{1 / 2 c \alpha}{\sqrt{1+\varepsilon_{3} \alpha^{2}}} \\
\frac{1 / 2 c \alpha}{\sqrt{1+\varepsilon_{3} \alpha^{2}}} & \frac{1+a \alpha+b \alpha^{2}}{1+\varepsilon_{3} \alpha^{2}}
\end{array}\right) .
$$

There exists a unitary transformation $u$ such that $u^{t} S^{\prime} u$ has the diagonal form $\operatorname{diag}\left(A^{\prime}, B^{\prime}\right)$. To prove that $\alpha$ may be chosen such that at least one of the coefficients on the diagonal is not 1 , it is enough to show that the determinant of $S^{\prime}$ is not equal to 1 in absolute value. We have

$$
\operatorname{det} S^{\prime}=\frac{1+a \alpha+\left(b-1 / 4 c^{2}\right) \alpha^{2}}{1+\varepsilon_{3} \alpha^{2}} .
$$

Since $a \neq 0$, clearly, $\left|\operatorname{det} S^{\prime}\right|$ cannot equal 1 for all $\alpha>0$ as $\alpha \rightarrow 0$. Thus we are in the situation of case (i) or (ii), and the extension to the origin follows.

Similarly, if in (30) $c \neq 0$, we consider the slice

$$
L=\left\{z \in \mathbb{C}^{n}: z_{4}=\cdots=z_{n}=0, z_{3}=\alpha z_{1}, \alpha>0\right\} .
$$

and $M \cap L$, in the diagonal form, will not have both coefficients of the harmonic part equal 1 for a suitable choice of $\alpha$.

Suppose now that $a=c=0$ and $b$ is arbitrary. Then consider the slice $L$ as in (32) but with $\alpha \in \mathbb{C} \backslash\{0\}$. We have

$$
M \cap L=\operatorname{Re}\left(z_{1}^{2}+\left(1+b \alpha^{2}\right) z_{2}^{2}\right)+\left|z_{1}\right|^{2}+\left(1+\varepsilon_{3}|\alpha|^{2}\right)\left|z_{2}\right|^{2} .
$$

In this case, the determinant of the corresponding matrix $S^{\prime}$ equals $\frac{1+b \alpha^{2}}{1+\varepsilon_{3}|\alpha|^{2}}$, which is not equal to 1 in absolute value for a suitable choice of $\alpha$.

The remaining case is when $\varepsilon_{j}=0$ for $j=3, \ldots, n$. Then the exists $z_{k}, k \neq 1,2$, such that the harmonic part of $M$ contains a monomial involving $z_{k}$. Without loss of generality we may assume $k=3$, and $M$ is defined by the equation

$$
\operatorname{Re}\left(z_{1}^{2}+z_{2}^{2}+a z_{2} z_{3}+b z_{3}^{2}+c z_{1} z_{3}+s(z)\right)+\left|z_{1}\right|^{2}+\left|z_{2}\right|^{2}=0,
$$

where at least one of $a, b$ and $c$ is non-zero. A similar analysis as above shows that for any choice of $a, b$ and $c$, there exists a slice $L$ of the form (32) or (37) such that 
$M \cap L$ is a quadratic cone in $\mathbb{C}^{2}$ with positive definite hermitian part such that the at least one of the coefficients of the harmonic part (when reduced to the diagonal form) is different from 1 . Thus we obtain the holomorphic extension to the origin. This proves the extension when the hermitian part of $M$ has at least two positive eigenvalues.

$\boldsymbol{\pi}=\boldsymbol{\nu}=\mathbf{1}$. Denote $z \in \mathbb{C}^{n}$ as $z=\left(\tilde{z}, z^{\prime}\right)$, where $\tilde{z}=\left(z_{1}, z_{2}\right) \in \mathbb{C}^{2}, z^{\prime}=\left(z_{3}, \ldots, z_{n}\right) \in$ $\mathbb{C}^{n-2}$. Any cone $M$ in $\mathbb{C}^{n}$ with $(\pi, \nu)=(1,1)$ can be written after a change of coordinates as

$$
\rho=\operatorname{Re}\left(\tilde{z}^{t} S \tilde{z}+2 z_{1} l_{1}\left(z^{\prime}\right)+2 z_{2} l_{2}\left(z^{\prime}\right)+q\left(z^{\prime}\right)\right)+\operatorname{Im}\left(z_{1} \overline{z_{2}}\right)=0,
$$

where $S \in \operatorname{Sym}(2, \mathbb{C}), l_{1}, l_{2}$ are $\mathbb{C}$-linear forms on $\mathbb{C}^{n-2}$ and $q$ is a complex quadratic form on $\mathbb{C}^{n-2}$. We consider the cases $q \equiv 0$ and $q \not \equiv 0$ separately.

$\boldsymbol{q} \equiv \mathbf{0}$. We begin by noting the following fact.

Lemma 6.1. Suppose that $M$ is given by (40) with $q \equiv 0$. Then there exists a coordinate system in which $M$ is given by

$$
\operatorname{Re}\left(\tilde{z}^{t} S \tilde{z}+2 R(z)\right)+\operatorname{Im}\left(z_{1} \overline{z_{2}}\right)=0,
$$

where $S \in \operatorname{Sym}(2, \mathbb{C})$, and $R$ is one of the following:

(i) 0 ,

(ii) $z_{1} z_{3}$,

(iii) $z_{2} z_{3}$,

(iv) $c z_{1} z_{3}+z_{2} z_{3}, c \in \mathbb{C} \backslash\{0\}$,

(v) $z_{1} z_{3}+z_{2} z_{4}$.

Proof. If $l_{1}=l_{2}=0$, we get (i). If $l_{2}=0$ but $l_{1} \neq 0$, then we can change coordinates in the $z^{\prime}$ variable to ensure that $l_{1}=z_{3}$, which gives us (ii). Similarly, if $l_{1}=0$ and $l_{2} \neq 0$ then we get (iii). If $l_{1}$ and $l_{2}$ are linearly dependent, but neither is 0 , we get (iv). Finally, if $l_{1}$ and $l_{2}$ are linearly independent, we can change coordinates in the last $n-2$ variables $z^{\prime}$ such that $l_{1}=z_{3}, l_{2}=z_{4}$, which gives us (v).

We will now consider the various possibilities for $R$.

(i) $R=0$. Then $M$ is given by $\operatorname{Re}\left(\tilde{z}^{t} S \tilde{z}\right)+\operatorname{Im}\left(z_{1} \overline{z_{2}}\right)=0$. This defining function involves only the variables $z_{1}$ and $z_{2}$. Let $L$ be the two dimensional subspace of $\mathbb{C}^{n}$ given by $\left\{z_{k}=0, k \geq 3\right\}$. Let $M^{\prime}=M \cap L$. $M^{\prime}$ can be identified with the cone in $\mathbb{C}^{2}$ given by $\operatorname{Re}\left(z^{t} S z\right)+\operatorname{Im}\left(z_{1} \overline{z_{2}}\right)=0$. Clearly $M^{\prime}$ has two-sided support, iff $M$ does. The result follows from the discussion at the beginning of this subsection (subsection 6.2).

(ii) $R=z_{1} z_{3}$. We let

$$
S=\left(\begin{array}{cc}
A & B \\
B & C
\end{array}\right)
$$

If $C=0$, the cone $M$ contains the complex hypersurface $\left\{z_{1}=0\right\}$, and therefore is non-minimal. If $C \neq 0$, we let $L$ be the two dimensional linear subspace of $\mathbb{C}^{n}$ given by

$$
\left\{\begin{array}{ccc}
z_{3} & = & \alpha z_{1}+\beta z_{2} \\
z_{k} & = & 0,
\end{array} \quad k \geq 4 .\right.
$$

Consider the two dimensional slice $M \cap L$, which is given by (43) and

$$
\operatorname{Re}\left(\tilde{z}^{t} S_{*} \tilde{z}\right)+\operatorname{Im}\left(z_{1} \overline{z_{2}}\right)=0,
$$


where

$$
S_{*}=\left(\begin{array}{cc}
A+2 \alpha & B+\beta \\
B+\beta & C
\end{array}\right) \text {. }
$$

If $\operatorname{Re}(C)=0$, we let

$$
\begin{aligned}
\alpha & =-\frac{1}{2}(A+C) \\
\beta & =-B+\sqrt{|C|^{2}+2}
\end{aligned} .
$$

Then $\operatorname{det} S_{*}=-2$. After a change of variables $z^{*}=e^{i \frac{\pi}{4}} z$, the equation of the cone in the new coordinates becomes $\operatorname{Re}\left(\tilde{z}^{t} \tilde{S} \tilde{z}\right)+\operatorname{Im}\left(z_{1} \overline{z_{2}}\right)=0$, where $\tilde{S}=i S_{*}$. Let $\tilde{S}=P+i Q$, with $P, Q$ real matrices. Then $\operatorname{det} \tilde{S}=2$, and $P=\operatorname{diag}(-i C, i C)$, so that $\operatorname{det} P=-(i C)^{2}<0$. Therefore, we can apply Corollary 6.1. If $\operatorname{Re}(C) \neq 0$, we let

$$
\begin{aligned}
\alpha & =-\frac{1}{2}(A+\bar{C}) \\
\beta & =-B+i \sqrt{|C|^{2}+2} .
\end{aligned}
$$

Then $\operatorname{det} S_{*}=2>1$. If $S_{*}=P+i Q$, where $P, Q \in \operatorname{Sym}(2, \mathbb{R})$, then $P=$ $\operatorname{diag}(-\operatorname{Re} C, \operatorname{Re} C)$, and $\operatorname{det} P=-(\operatorname{Re} C)^{2}<0$. Again, we can apply Corollary 6.1.

(iii) $R=z_{2} z_{3}$. This is similar to case (ii).

(iv) $R=c z_{1} z_{3}+z_{2} z_{3}, c \in \mathbb{C} \backslash\{0\}$. If $c \in \mathbb{R} \backslash\{0\}$, the linear change of variables $\left(w_{1}, w_{2}, w^{\prime}\right)=\left(c z_{1}+z_{2}, \frac{1}{c} z_{2}, z^{\prime}\right)$ reduces the situation to case (ii). Observe that the matrix corresponding to this change of coordinates in the $z_{1}, z_{2}$ variables belongs to $S L(2, \mathbb{R})$, and therefore preserves the hermitian part $\operatorname{Im}\left(z_{1} \overline{z_{2}}\right)$. (Lemma $\left.4.1(\mathrm{a})\right)$.

If $c \in \mathbb{C} \backslash \mathbb{R}$, then $M$ is given by

$$
\operatorname{Re}\left(\tilde{z}^{t} S \tilde{z}+2\left(c z_{1}+z_{2}\right) z_{3}\right)+\operatorname{Im}\left(z_{1} \overline{z_{2}}\right)=0,
$$

where $S$ is as in (42). For $\alpha \in \mathbb{C} \backslash\{0\}$, let $L_{\alpha} \subset \mathbb{C}^{n}$ be the two dimensional subspace given by

$$
\left\{\begin{array}{l}
z_{3}=\quad \alpha z_{2} \\
z_{k}=0, k \geq 4 .
\end{array}\right.
$$

Then $M \cap L_{\alpha}$ is given by the above equations and the equation $\operatorname{Re}\left(\tilde{z}^{t}(S+\alpha T) \tilde{z}\right)+$ $\operatorname{Im}\left(z_{1} \overline{z_{2}}\right)=0$, where

$$
T=\left(\begin{array}{ll}
0 & c \\
c & 2
\end{array}\right) .
$$

We set $S_{*}=S+\alpha T$, and let $\zeta$ be such that

$$
\zeta^{4}=\frac{\overline{\operatorname{det} S_{*}}}{\left|\operatorname{det} S_{*}\right|} .
$$

After a change of coordinates $z^{*}=\zeta z$, the cone is represented by $\operatorname{Re}\left(\tilde{z}^{t} \tilde{S} \tilde{z}\right)+$ $\operatorname{Im}\left(z_{1} \overline{z_{2}}\right)=0$, where $\tilde{S}=\zeta^{2} S_{*}$. By the choice of $\zeta$, $\operatorname{det} \tilde{S}>0$. We now obtain an asymptotic expression for $\zeta^{2}$ as $|\alpha| \rightarrow \infty$ :

$$
\begin{aligned}
\pm \zeta^{2} & =\sqrt{\frac{\overline{A C}+2 \bar{A} \bar{\alpha}-(\bar{B}+\overline{c \alpha})^{2}}{\left|A C+2 A \alpha-(B+c \alpha)^{2}\right|}} \\
& =i \frac{\bar{\alpha}}{|\alpha|} \cdot \frac{\bar{c}}{|c|}+O\left(\frac{1}{|\alpha|}\right) .
\end{aligned}
$$

Therefore, as $|\alpha| \rightarrow \infty$,

$$
\pm \alpha \zeta^{2}=i|\alpha| \frac{\bar{c}}{|c|}+O(1)
$$


and

$$
\pm \operatorname{Re}\left(\alpha \zeta^{2}\right)=|\alpha| \sin (\arg c)+O(1) .
$$

Let $\theta$ be such that $\sin (\arg c)$ and $\sin (\theta+\arg c-\arg A)$ have different signs. (Note that since $c \in \mathbb{C} \backslash \mathbb{R}, \sin (\arg c) \neq 0$.) Let $\alpha=|\alpha| e^{i \theta}$, where $|\alpha|$ is large and will be chosen later. Using (47) again,

$$
\pm \operatorname{Re}\left(A \zeta^{2}\right)=|A| \sin (\theta+\arg c-\arg A)+O\left(\frac{1}{|\alpha|}\right) .
$$

We now show that $|\alpha|$ can be chosen such that $\operatorname{det} \tilde{S}>1$ and if $\tilde{S}=P+i Q$ (with $P, Q$ real), then $\operatorname{det} P<0$. A computation shows that

$$
\operatorname{det} P=\operatorname{Re}\left(A \zeta^{2}\right) \operatorname{Re}\left(C \zeta^{2}\right)+2 \operatorname{Re}\left(A \zeta^{2}\right) \operatorname{Re}\left(\alpha \zeta^{2}\right)-\left(\operatorname{Re}\left((B+C \alpha) \zeta^{2}\right)\right)^{2} .
$$

From (48) and (49) above, we see that there is $K>0$ such that for $|\alpha|$ large enough

$$
\operatorname{Re}\left(A \zeta^{2}\right) \operatorname{Re}\left(\alpha \zeta^{2}\right)<-K|\alpha| .
$$

Since the first term in the right in (50) is bounded by $|A||C|$, it follows that we can choose $|\alpha|$ so large that $\operatorname{det} P<0$. Also $\operatorname{det} \tilde{S} \rightarrow \infty$ as $|\alpha| \rightarrow \infty$, it follows that we can also make $\operatorname{det} \tilde{S}>\frac{1}{4}$. The result now follows from Corollary 6.1]

(v) $R=z_{1} z_{3}+z_{2} z_{4}$. If $A \neq 0$ or $C \neq 0$, the situation can be clearly reduced to (ii) or (iii). Therefore, the only new situation is when $A=C=0$, i.e.,

$$
S=\left(\begin{array}{cc}
0 & B \\
B & 0
\end{array}\right) \text {. }
$$

Let $L \subset \mathbb{C}^{n}$ be the two dimensional linear subspace defined by

$$
\left\{\begin{array}{c}
z_{3}=\frac{1}{2} z_{1}+\left(-\frac{B}{2}+i\right) z_{2} \\
z_{4}=\left(-\frac{B}{2}+i\right) z_{1}-\frac{1}{2} z_{2} \\
z_{k}=0, k \geq 5
\end{array}\right.
$$

Then $M \cap L$ is given by the above equations and

$$
\operatorname{Re}\left(\tilde{z}^{t} S_{*} \tilde{z}\right)+\operatorname{Im}\left(z_{1} z_{2}\right)=0,
$$

where

$$
S_{*}=\left(\begin{array}{cc}
1 & 2 i \\
2 i & -1
\end{array}\right) .
$$

It follows that $\operatorname{det} S_{*}=3$, and if $S_{*}=P+i Q$, then $P=\operatorname{diag}(1,-1)$, so that $\operatorname{det} P=-1<0$. Again, extension follows from Corollary 6.1

$\boldsymbol{q} \not \equiv \mathbf{0}$. In the $\tilde{z}=\left(z_{1}, z_{2}\right)$ variable, we make the change (20), and in the $z^{\prime}=$ $\left(z_{3}, \ldots, z_{n}\right)$, we make a linear change of variables so that $M$ is given by

$$
\operatorname{Re}\left(\tilde{z}^{t} S \tilde{z}+2 z_{1} l_{1}\left(z^{\prime}\right)+2 z_{2} l_{2}\left(z^{\prime}\right)+\sum_{j=3}^{k} z_{j}^{2}\right)+\left|z_{1}\right|^{2}-\left|z_{2}\right|^{2},
$$

where $l_{1}$ and $l_{2}$ are complex linear functionals on $\mathbb{C}^{n-2}$, and $3 \leq k \leq n$. Now let $L$ be given by

$$
z_{k}=0 \text { for } k \geq 4 .
$$

Then $M \cap L$ is given by the equations (51) and

$$
\operatorname{Re}\left(\tilde{z}^{t} S \tilde{z}+2 c_{1} z_{1} z_{3}+2 c_{2} z_{2} z_{3}+z_{3}^{2}\right)+\left|z_{1}\right|^{2}-\left|z_{2}\right|^{2}=0,
$$


where $c_{1}, c_{2} \in \mathbb{C}$. Define

$$
\left\{\begin{array}{l}
z_{3}^{*}=c_{1} z_{1}+c_{2} z_{2}+z_{3} \\
z_{k}^{*}=z_{k}, \text { for } k \neq 4
\end{array} .\right.
$$

With respect to the new coordinates (after suppressing the asterisk for convenience), $M \cap L$ is given by equations (51) along with

$$
\operatorname{Re}\left(\tilde{z}^{t} S^{\prime} \tilde{z}+z_{3}^{2}\right)+\left|z_{1}\right|^{2}-\left|z_{2}\right|^{2}=0
$$

where $S^{\prime} \in \operatorname{Sym}(2, \mathbb{C})$. Assume that

$$
S=\left(\begin{array}{ll}
A & B \\
B & C
\end{array}\right)
$$

and let $L_{\alpha} \subset L$ be the two dimensional linear subspace of $\mathbb{C}^{3}$ defined by

$$
z_{2}=\alpha z_{1}
$$

where $\alpha$ is a complex number such that $|\alpha| \neq 1$. Then $M \cap L_{\alpha}$ is given by (51), (52) and

$$
\operatorname{Re}\left(\left(A+2 B \alpha+C \alpha^{2}\right) z_{1}^{2}+z_{3}^{2}\right)+\left(1-|\alpha|^{2}\right)\left|z_{1}\right|^{2}=0 .
$$

After rescaling the $z_{1}$ coordinate, $M \cap L_{\alpha}$ is represented by (51), (52), and

$$
\operatorname{Re}\left(\left|E_{\alpha}\right| z_{1}^{2}+z_{3}^{2}\right)+\left|z_{1}\right|^{2}=0, \text { where } E_{\alpha}=\frac{A+2 B \alpha+C \alpha^{2}}{1-|\alpha|^{2}} \text {. }
$$

Repeating the construction of the family of analytic discs for type $\mathcal{M}_{(1,0)}^{1}$, we prove that for this cone holomorphic functions on one side have extension to the origin.

$\boldsymbol{\pi}=\mathbf{1}, \boldsymbol{\nu}=\mathbf{0}$. We can write $M$ as

$$
\operatorname{Re}\left(A z_{1}^{2}+z_{1} l\left(z^{\prime}\right)+q\left(z^{\prime}\right)\right)+\left|z_{1}\right|^{2}=0
$$

where $z^{\prime} \in \mathbb{C}^{n-1}, l$ and $q$ are complex linear and quadratic forms on $\mathbb{C}^{n-1}$ respectively. Note that if $q \equiv 0$, we have $\left\{z_{1}=0\right\} \subset M$, so that $M$ is non-minimal. We will therefore assume that $q \neq 0$.

If $l \equiv 0$, by a linear change of variables in $z^{\prime}$, we can assume that $q\left(z^{\prime}\right)=\sum_{j=2}^{k} z_{j}^{2}$, where $2 \leq k \leq n$. Taking a slice by $L=\left\{z_{k}=0, k \geq 3\right\}$ we see that $M \cap L$ is of type $\mathcal{M}_{(1,0)}^{1}$, which does not have two-sided support.

If $l \neq 0$, we can assume that $l\left(z^{\prime}\right)=z_{2}$ after a change of variables. If $\frac{\partial q}{\partial z_{2}} \neq 0$, then we can again take a slice by $L=\left\{z_{k}=0, k \geq 3\right\}$, and again we see that $M \cap L$ is of type $\mathcal{M}_{(1,0)}^{1}$.

If $\frac{\partial q}{\partial z_{2}}=0$, we can change variables in the $\left(z_{3}, \ldots, z_{n}\right)$ variables to diagonalize $q$ and write $q\left(z^{\prime}\right)=\sum_{j=3}^{k} z_{j}^{2}$, where $3 \leq k \leq n$. We take a slice by $L=\left\{z_{k}=\right.$ $0, k=2$, or $k \geq 4\}$. Then $M \cap L$ is a cone of type $\mathcal{M}_{(1,0)}$ in the $z_{1} z_{3}$ plane. This completes the proof for $\pi=1, \nu=0$.

$\boldsymbol{\pi}=\boldsymbol{\nu}=\mathbf{0}$. These cones are clearly non-minimal. Theorem 1.1 is proved. 


\section{Classification of quadratic Cones With two-Sided support.}

As a corollary to the proof of Theorem 1.1 we obtain the following complete classification of real quadratic cones in $\mathbb{C}^{n}$ which admit two-sided support by complex hypersurfaces.

Proposition 7.1. Let $M \subset \mathbb{C}^{n}$ be a real quadratic cone in $\mathbb{C}^{n}$ which admits twosided support at 0 . Then one of the following holds.

(i) $M$ is biholomorphic to $M^{\prime} \times \mathbb{C}^{n-2}$, where $M^{\prime}$ has the normal form either of type $\mathcal{M}_{(1,1)}^{1}$ with $0 \leq B \leq A \leq 1$, or $A=B$, or one of $\mathcal{M}_{(1,1)}^{2}, \mathcal{M}_{(1,1)}^{3}, \mathcal{M}_{(1,1)}^{4}$, $\mathcal{M}_{(1,0)}^{2}$, or $\mathcal{M}_{(0,0)}^{1}$.

(ii) $M$ is biholomorphic either to

$$
\left\{\operatorname{Re}\left(z_{1}^{2}+\cdots+z_{k}^{2}\right)=0,2<k \leq n\right\}
$$

or

$$
\left\{\operatorname{Re}\left(z_{1} z_{2}+z_{1} \overline{z_{3}}\right)=0\right\}
$$

Proof. (i) If $n=2$, this follows immediately from Section 6.1. If $n \geq 3$, it is clear that $M=M^{\prime} \times \mathbb{C}^{n-2}$ has two-sided support iff $M^{\prime}$ has two-sided support in $\mathbb{C}^{2}$, so the result follows in this case as well.

(ii) An examination of the proof in Section 6.2 shows that every cone in three variables, which is not extendable, is, in fact, non-minimal. Let $A=\{\alpha=0\} \subset M$ be the germ of an irreducible complex hypersurface at the origin. Then, for the tangent cones, $T_{0} A \subset T_{0} M=M$, but since $A$ is a complex hypersurface, $T_{0} A=$ $\left\{\alpha_{\mu}=0\right\}$, where $\alpha_{\mu}$ is the homogeneous polynomial consisting of the nonzero terms of the least degree in the Taylor expansion of $\alpha$. It follows that we can take $\alpha$ to be a homogeneous complex polynomial, and since $\rho$ has degree $2, \alpha$ has degree 1 or 2 . Therefore, there is a real polynomial $\varphi$ on $\mathbb{C}^{n}$ such that $\rho=\varphi \alpha+\overline{\varphi \alpha}=\operatorname{Re}(\varphi \alpha)$. If $\rho$ is of degree $2, \varphi$ has degree 0 , and $\rho=\operatorname{Re}(c \alpha)$ for some constant $c$. After a linear change of variables, we can diagonalize the complex quadratic form $c \alpha$, and obtain (53). If $\operatorname{deg} \alpha=\operatorname{deg} \varphi=1$, then $\varphi=\lambda+\bar{\mu}$, where $\lambda$ and $\mu$ are complex linear maps from $\mathbb{C}^{n}$ to $\mathbb{C}$. First suppose that $\{\alpha, \varphi, \mu\}$ is a linearly independent set in the dual a space of $\mathbb{C}^{n}$. We can find a system of coordinates $\left(z_{1}, \ldots, z_{n}\right)$ on $\mathbb{C}^{n}$ such that $z_{1}=\alpha, z_{2}=\lambda$ and $z_{3}=\mu$. Therefore, we have $\rho=\operatorname{Re}(\varphi \alpha)=\operatorname{Re}\left(\left(z_{2}+\overline{z_{3}}\right) z_{1}\right)$, which gives (54). If $\alpha, \lambda, \mu$ are linearly dependent, we are reduced to classifying non-minimal cones in $\mathbb{C}^{2}$.

\section{TWO-SIDED SUPPORT FOR SMOOTH HYPERSURFACES: PROOF OF Proposition 1.1.}

Proposition 1.1 will be deduced from the following lemma, for which we do not claim any originality. For completeness, we give a proof essentially following [21, Lem. 3.2].

Lemma 8.1. Let $\Omega \subset \mathbb{C}^{n}$ be a domain whose boundary can be locally represented as a Lipschitz graph and let $p \in \partial \Omega$. Suppose that there is an open set $U \subset \mathbb{C}^{n}$ containing $p$ and a holomorphic function $f \in \mathcal{O}(\Omega \cap U)$ which does not extend to any neighbourhood of $p$, and let $A$ be a germ at $p$ of complex analytic sets such that $A \subset \bar{\Omega}$. Then $A \subset \partial \Omega$. 
Proof. Since the problem is local, we can assume that the coordinates $\left(z^{\prime}, z_{n}\right) \in$ $\mathbb{C}^{n-1} \times \mathbb{C}$ have been so chosen that $p=0$, and

$$
\Omega=\left\{z \in \mathbb{C}^{n},\left(z^{\prime}, x_{n}\right) \in U: y_{n}>g\left(z^{\prime}, x_{n}\right)\right\}
$$

where $g$ is a real-valued Lipschitz function defined in a convex neighbourhood $U$ of 0 in $\mathbb{C}^{n-1} \times \mathbb{R}$. It is known [21, [10] that the envelope of holomorphy $\widehat{\Omega}$ of $\Omega$ is schlicht and of the form

$$
\widehat{\Omega}=\left\{z \in \mathbb{C}^{n},\left(z^{\prime}, x_{n}\right) \in U: y_{n}>\hat{g}\left(z^{\prime}, x_{n}\right)\right\},
$$

where $\hat{g} \leq g$ is an upper semi-continuous function. Since the origin is a point of non-extendability, it follows that $\hat{g}(0,0)=g(0,0)=0$.

By a standard abuse of notation, denote by $A$ a particular representative of the germ $A$ at the origin. Without loss of generality we may assume that $A$ is irreducible. Arguing by contradiction, suppose that there exists $w \in A \backslash \partial \Omega$ arbitrarily close to the origin. Let $A_{w}$ be an irreducible complex curve in $A$ connecting $w$ and the origin. Note that such $A_{w}$ always exists. Indeed, if $\operatorname{dim} A=1$, then $A_{w}=A$, so assume that $\operatorname{dim} A=d \geq 2$. Then near the origin $A$ can be represented as a branched covering $\pi: A \rightarrow L$ over a $d$-dimensional subspace $L \subset \mathbb{C}^{n}$. Further, in some neighbourhood $V$ of the origin, $\pi^{-1}(0)=0$. We may assume that $w \in V$. Let $l \subset L$ be the complex line connecting $\pi(w)$ and the origin. Then one of the irreducible components of $\pi^{-1}(l)$ is the desired curve.

Using a Puiseux parametrization we obtain a holomorphic map $\varphi$ from the closed unit disc $\Delta$ into $\mathbb{C}^{n}$ such that $\varphi(\Delta)$ is a neighbourhood of 0 in $A_{w}$, and $\varphi(\Delta) \subset$ $\bar{\Omega} \cap U \cap V$, where $U$ is a neighbourhood of 0 as in the statement of the lemma, i.e., some $f \in \mathcal{O}(\Omega \cap U)$ does not extend to any neighbourhood of 0 , and $V$ is a neighbourhood of the origin such that there is a constant $C \geq 1$ such that for $z \in V$,

$$
\operatorname{dist}(z, \partial \Omega) \geq C^{-1}\left(y_{n}-g\left(z^{\prime}, x_{n}\right)\right) .
$$

Such $V$ exists since $\partial \Omega$ is Lipschitz.

Applying an automorphism of $\Delta$, we can assume that $\varphi(0)=0$. Let $\chi(z)=$ $-\log \operatorname{dist}(z, \partial \Omega)$ and $\hat{\chi}(z)=-\log \operatorname{dist}(z, \partial \widehat{\Omega})$. Then $\hat{\chi}$ is a plurisubharmonic exhaustion of $\widehat{\Omega}$, and on $\Omega$ we have $\chi \leq \hat{\chi}$. For $t>0$ and $\zeta \in \Delta$ define

$$
\psi_{t}(\zeta)=\hat{\chi}\left(\varphi(\zeta)+t i e_{n}\right),
$$

where $e_{n}=(0, \ldots, 0,1)$ is the $n$-th standard basis element of $\mathbb{C}^{n}$. Then $\psi_{t}$ is a subharmonic function for each $t$. Further, define for $k>0$

$$
\mu_{k}(t)=\text { Lebesgue measure of }\left\{\zeta \in \Delta: \psi_{t}(\zeta)<k\right\} .
$$

To prove our result it is sufficient to show that for all $k$ we have $\lim _{t \rightarrow 0^{+}} \mu_{k}(t)=0$. We claim that for small $t$,

$$
\psi_{t}(0) \geq \log \left(\frac{1}{t}\right)
$$

and

$$
\max _{\zeta \in \Delta}\left(\psi_{t}(\zeta)\right) \leq \log \frac{C}{t}
$$

where $C$ is as in 55 .

For (56) note that since $0 \in \partial \widehat{\Omega}$, we have $\operatorname{dist}\left(\right.$ ite $\left._{n}, \partial \widehat{\Omega}\right) \leq \operatorname{dist}\left(\right.$ ite $\left._{n}, 0\right)=t$, so $\psi_{t}(0)=-\log \operatorname{dist}\left(\right.$ ite $\left._{n}, \partial \widehat{\Omega}\right) \geq-\log t$. 
For (57) observe that there is a nonempty subset $T \subset \partial \Delta$ such that $\varphi(T) \subset \partial \Omega$ (otherwise by the Kontinuitätssatz $f$ would extend to a neighbourhood of 0.) From (55),

$$
\begin{aligned}
\operatorname{dist}\left(z+i t e_{n}, \partial \Omega\right) & \geq \frac{1}{C}\left(y_{n}+t-g\left(z^{\prime}, x_{n}\right)\right) \\
& =\frac{1}{C}\left(y-g\left(z^{\prime}, x_{n}\right)\right)+\frac{t}{C}
\end{aligned}
$$

In particular, if $z \in \partial \Omega$, then $\operatorname{dist}\left(z+i t e_{n}, \partial \Omega\right) \geq \frac{t}{C}$. Since $\varphi(\zeta) \in \partial \Omega$ for $\zeta \in T$, we have for small $t$,

Therefore,

$$
\min _{\zeta \in \partial \Delta} \operatorname{dist}\left(\varphi(\zeta)+i t e_{n}, \partial \Omega\right) \geq \frac{t}{C}
$$

$$
\begin{aligned}
\max _{\zeta \in \Delta}\left(\psi_{t}(\zeta)\right) & =\max _{\zeta \in \partial \Delta} \hat{\chi}\left(\varphi(\zeta)+i t e_{n}\right) \\
& \leq \max _{\zeta \in \partial \Delta} \chi\left(\varphi(\zeta)+i t e_{n}\right) \\
& =-\log \min _{\zeta \in \partial \Delta} \operatorname{dist}\left(\varphi(\zeta)+i t e_{n}, \partial \Omega\right) \\
& \leq-\log \frac{t}{C},
\end{aligned}
$$

which proves the second claim. Now apply the sub-averaging property to the subharmonic function $\psi_{t}$ :

$$
\begin{aligned}
\pi \log \left(\frac{1}{t}\right) & \leq \pi \psi_{t}(0) \\
& \leq \int_{\Delta} \psi_{t}(\zeta) d \xi d \eta \\
& =\int_{\left\{\psi_{t}(\zeta)<k\right\}}+\int_{\left\{\psi_{t}(\zeta) \geq k\right\}} \\
& \leq k \mu_{k}(t)+\left(\pi-\mu_{k}(t)\right) \max _{\zeta \in \Delta} \psi_{t}(\zeta) \\
& \leq k \mu_{k}(t)+\left(\pi-\mu_{k}(t)\right) \log \left(\frac{C}{t}\right) \\
& =\left(k-\log \left(\frac{C}{t}\right)\right) \mu_{k}(t)+\pi \log \left(\frac{C}{t}\right)
\end{aligned}
$$

Therefore, for small $t$, we have:

$$
\begin{aligned}
\mu_{k}(t) & \leq \frac{\pi\left[\log \left(\frac{1}{t}\right)-\log \frac{C}{t}\right]}{k-\log C+\log t} \\
& =\frac{-\pi \log C}{k-\log C+\log t} \\
& \rightarrow 0 \text { as } t \rightarrow 0^{+}
\end{aligned}
$$

Proof of Proposition 1.1. Let $A^{-}$be defined in a neighbourhood $U$ of $p$ in $\mathbb{C}^{n}$ by an equation $\{z \in U: f(z)=0\}$. Then $\frac{1}{f} \in \mathcal{O}\left(\Omega^{+} \cap U\right)$ does not extend to any neighbourhood of $p$. Since $A^{+} \subset \bar{\Omega}$, by Lemma 8.1, $A^{+} \subset M$. By the same argument, $A^{-} \subset M$. If $A^{+} \neq A^{-}$, then $A=A^{+} \cup A^{-}$is a non-smooth complex 
hypersurface through $p$, and $A \subset M$. But any complex hypersurface contained in a hypersurface represented as a graph is smooth (See [12, Lemma 3].)

\section{REFERENCES}

[1] Aldo Andreotti and C. Denson Hill. E. E. Levi convexity and the Hans Lewy problem. I. Reduction to vanishing theorems. Ann. Scuola Norm. Sup. Pisa (3) 26 (1972), 325-363.

[2] M. Salah Baouendi and Linda Preiss Rothschild. Cauchy-Riemann functions on manifolds of higher codimension in complex space. Invent. Math. 101 (1990), no. 1, 45-56.

[3] M. Salah Baouendi and F. Trèves. A property of the functions and distributions annihilated by a locally integrable system of complex vector fields. Ann. of Math. (2) 113 (1981), no. 2, $387-421$.

[4] Eric Bedford and John Erik Fornæss. Local extension of CR functions from weakly pseudoconvex boundaries. Michigan Math. J., 25 (1978), no. 3, 259-262.

[5] Daniel Burns and Xianghong Gong. Singular Levi-flat real analytic hypersurfaces. Amer. J. Math., 121 (1999), no. 1, 23-53.

[6] Debraj Chakrabarti and Rasul Shafikov. Holomorphic extension of CR functions from quadratic cones. Math. Ann. 341, (2008), 543-573.

[7] Debraj Chakrabarti and Rasul Shafikov. Erratum: Holomorphic extension of CR functions from quadratic cones. Math. Ann. 345 (2009), 491-492.

[8] E. M Cirka. Analytic representation of CR-functions. Mat. Sb. (N.S.) 98 (140) (1975), no. 4(12), 591-623, 640. English translation in Math. USSR Sbornik 27(1975), No.4, 526-553.

[9] E. M. Chirka. Complex analytic sets, volume 46 of Mathematics and its Applications (Soviet Series). Kluwer Academic Publishers Group, Dordrecht, 1989.

[10] E. M. Chirka. Levi and Trépreau theorems for continuous graphs. Tr. Mat. Inst. Steklova 235 (2001), Anal. i Geom. Vopr. Kompleks. Analiza, 272-287; translation in Proc. Steklov Inst. Math. 235 (2001), no. 4, 261-276.

[11] E. M. Chirka and Edgar Lee Stout. Removable singularities in the boundary. Contributions to complex analysis and analytic geometry, Aspects Math., E26, Vieweg, Braunschweig, 1994, p. $43-104$.

[12] E. M. Chirka. Rado's theorem for CR-mappings of hypersurfaces. Mat. Sb., 185, (1994), no. 6, 125-144. English translation in Russian Acad. Sci. Sb. Math.82 (1995), no. 1, 243-259.

[13] Ju. B. Ermolaev. The simultaneous reduction of symmetric and Hermitian forms. Izv. Vyš̌. Učebn. Zaved. Matematika, (1961) no. 2 (21), 10-23.

[14] Herbert Federer. Geometric measure theory. Die Grundlehren der mathematischen Wissenschaften, Band 153 Springer-Verlag New York Inc., New York 1969.

[15] J. J. Kohn and L. Nirenberg. A pseudo-convex domain not admitting a holomorphic support function. Math. Ann., 201 (1973), 265-268.

[16] A. Kytmanov, S. Myslivets and N. Tarkhanov. Removable singularities of CR functions on singular boundaries. Math. Z. 242 (2002), no. 3, 491-515.

[17] Joël Merker and Edmont Porten. Holomorphic extension of CR functions, envelopes of holomorphy, and removable singularities. Int. Math. Res. Surv. 2006, 1-287.

[18] Jean-Pierre Rosay. À propos de "wedges" et d' "edges", et de prolongements holomorphes. Trans. Amer. Math. Soc. 297 (1986), no. 1, 63-72.

[19] N. V. Shcherbina. On the polynomial hull of a graph. Indiana Univ. Math. J. 42 (1993), no. $2,477-503$.

[20] T. Takagi. On an algebraic problem related to an analytic theorem of Carathéodory and Fejér and on an allied theorem of Landau. Japan J. Math. 1 (1924) 83-93. Also reprinted in his Collected Works, Springer-Verlag Tokyo, 1990, pp. 226-234.

[21] J.-M. Trépreau. Sur le prolongement holomorphe des fonctions C-R défines sur une hypersurface réelle de classe $C^{2}$ dans $\mathbf{C}^{n}$. Invent. Math., 83 (1986), no. 3,583-592.

[22] A.E. Tumanov Extension of CR-functions into a wedge from a manifold of finite type. Mat. Sb. (N.S.) 136(178) (1988), no. 1, 128-139; translation in Math. USSR-Sb. 64 (1989), no. $1,129-140$. 